\title{
Pediatric Chest Disorders: Practical Imaging Approach to Diagnosis
}

\author{
Alison Hart and Edward Y. Lee
}

\section{Learning Objectives}

- To apply an anatomical three-compartment model to some of the most commonly encountered pediatric chest pathologies including primary processes of the lung parenchyma, abnormalities of the large airway, and pathology originating in the mediastinum

- To understand the advantages and disadvantages of current imaging modalities in imaging of the pediatric chest

\subsection{Introduction}

Symptomatology referable to the chest is one of the most common reasons for which pediatric patients present for clinical evaluation. While subsequent diagnosis widely varies depending upon the specific symptoms, clinical context, and age of the patient, imaging plays a critical role in elucidation of differential considerations. Furthermore, imaging provides valuable information in the evaluation of disease extent and associated abnormalities thereby directly impacting patient management decisions. Furthermore, diagnostic imaging provides a detailed assessment of treatment response.

Given the broad range of pathologies which affect the pediatric chest, anatomic localization is a useful classification approach and can be thought of in a three-compartment model, including primary processes of the lung parenchyma, abnormalities of the large airway, and pathology originating in the mediastinum. Here, we present this practical approach as it applies to some of the most commonly encountered

\footnotetext{
A. Hart · E. Y. Lee $(\square)$

Department of Radiology, Boston Children's Hospital and Harvard Medical School, Boston, MA, USA

e-mail: Edward.Lee@childrens.harvard.edu
}

pediatric chest pathologies. This chapter also reviews the advantages and disadvantages of current imaging modalities and characteristic imaging findings of pediatric thoracic disorders encountered in daily clinical practice.

\subsection{Advantages and Disadvantages of Imaging Modalities}

Three most commonly used imaging modalities for evaluating pediatric thoracic disorders are chest radiography, computed tomography (CT), and magnetic resonance imaging (MRI). Their advantages and disadvantages are briefly summarized in the following section.

\subsubsection{Chest Radiography}

Chest radiography is the first-line and most commonly performed imaging examination in pediatric patients with potential thoracic disorders. Frequently, it is also the sole imaging study required for diagnosis. In thoracic pathologies unable to be fully characterized by chest radiography alone, such as those involving mediastinum or airway, it is often sufficient for appropriately tailoring subsequent imaging examinations. Given that ionizing radiation is required for the generation of chest radiography, its use must be weighed against the potential risk factor of radiation-induced malignancy. This is heightened in pediatric patients due to their increased susceptibility to the effects of ionizing radiation as well as their longer potential for repeated exposure as compared with adults. In the implementation of the ALARA principle, whereby radiation levels are maintained as low as reasonably achievable (ALARA), diagnostic chest radiography can be generated with very low radiation dose [1, 2]. This, thus, translates into a very low risk of the development of radiation-induced malignancy. Additionally, chest radiography offers the advantages of easy acquisition, low cost, and ready availability. 


\subsubsection{Computed Tomography}

$\mathrm{CT}$ is the most common cross-sectional examination utilized in the evaluation of abnormalities of the lung parenchyma. This is due to its superior anatomic resolution and the high contrast between lung pathology and the adjacent air-filled lung parenchyma, a difference which can be further augmented with the use of intravenous contrast. Compared with chest radiography, $\mathrm{CT}$ provides superior characterization of abnormalities and aids in preprocedural evaluation by affording superior relational anatomy. Specialized CT techniques such as high-resolution acquisition, inspiratory and expiratory sequences, and volumetric 3D/4D image reconstruction can be used to answer specific clinical questions [3]. The rapid acquisition time afforded by the multidetector $\mathrm{CT}$ (MDCT) scanners currently in use is of further benefit, especially in the pediatric population, because it can diminish the need for sedation. While the benefits of CT are numerous, the most glaring disadvantage is the added ionizing radiation incurred over radiography, and, thus, the risk to benefit ratio must be weighed carefully. Tailored image acquisition parameters which account for patient body size as well as image optimization techniques such as iterative reconstruction in accordance with the principal of ALARA must be used for dose reduction [4].

\subsubsection{Magnetic Resonance Imaging}

MRI affords superior soft tissue contrast as compared to CT. Therefore, it is an ideal modality for characterization of extra-parenchymal soft tissue lesions such as those of the mediastinum or within the soft tissues of the chest wall. The lack of ionizing radiation, as compared with $\mathrm{CT}$, is of added benefit especially in susceptible pediatric patients. Use of MRI in the evaluation of the lung parenchyma is hindered by the intrinsic paucity of photons within the air-filled alveoli which results in a small magnitude of MRI signal generation [5]. This generated signal is further degraded by increased susceptibility artifact which results from the many air-tissue interfaces within the lung. The decreased special resolution of MRI as compared with CT further limits its use in the evaluation of subtle pathologic changes of the lung parenchyma [6]. As such, MRI is of limited utility in the evaluation of lung parenchymal pathologies which result in an increase of air, the so-called negative pathologies, such as cystic lung disease or emphysema. However, recent development of improved MRI sequences has increased utilization of MRI for evaluation of the so-called plus pathologies which result in the deposition of material within the lung parenchyma [7, 8]. Because of this additive process, a larger number of photons are present within the lung parenchyma thereby increasing the magnitude of the MR signal generated. Disadvantages of
MRI include availability and cost. In addition, because of long acquisition times, sedation is usually required as patient motion, in addition to the respiratory and cardiac motion intrinsic to imaging of the chest, can significantly degrade image quality and interfere with image interpretation.

\subsection{Spectrum of Pediatric Thoracic Disorders}

\subsubsection{Pediatric Lung Disorders}

\subsubsection{Neonatal Lung Disorders}

\section{Surfactant Deficiency Syndrome due to Prematurity}

Surfactant deficiency syndrome is the most common cause of death in premature infants, affecting 6.1/1000 live births [9], with incidence inversely related to gestational age. A lipoprotein produced by type II pneumocytes, surfactant production begins at 23 weeks gestation and maturation occurs at 35 weeks gestation [10]. Allowing for full opening of lung alveoli by reducing alveolar surface tension, surfactant increases lung compliance. In the absence of surfactant, lung compliance decreases leading to atelectasis and alveolar damage. Alveolar destruction results in the generation of cellular debris and fibrin which combine to form hyaline membranes which in turn impair oxygen exchange. Besides prematurity, other risk factors for developing surfactant deficiency syndrome include male gender, cesarean delivery, multiple gestations, and maternal diabetes due to decreased surfactant maturation at high serum insulin levels. Within minutes of birth, affected infants typically develop symptoms including grunting, nasal flaring, retractions, tachypnea, and cyanosis.

Chest radiography is the main imaging modality for evaluating surfactant deficiency syndrome [10] (Fig. 10.1). It classically demonstrates low lung volumes with diffuse fine granular/reticulogranular opacities or, in more severe cases, complete lung opacity. Air bronchograms may be variably present. In the absence of treatment, imaging findings progress reproducibly, being most severe at birth and normalizing over the course of days to weeks or progressing to chronic lung disease of prematurity (Fig. 10.2a). CT is infrequently utilized in surfactant deficiency syndrome but may help in the evaluation of long-term sequela (Fig. 10.2b).

Three currently available treatments include [1] delaying labor to allow for surfactant maturation to continue in utero, [2] administering antenatal maternal glucocorticoids which cross the placenta and stimulate surfactant production, and [3] providing surfactant replacement therapy at birth either in aerosolized or nebulized form. Although treatment advances have significantly decreased mortality from surfactant deficiency as a result of prematurity, the archetypal radiographic features have changed. Mechanical ventilator 


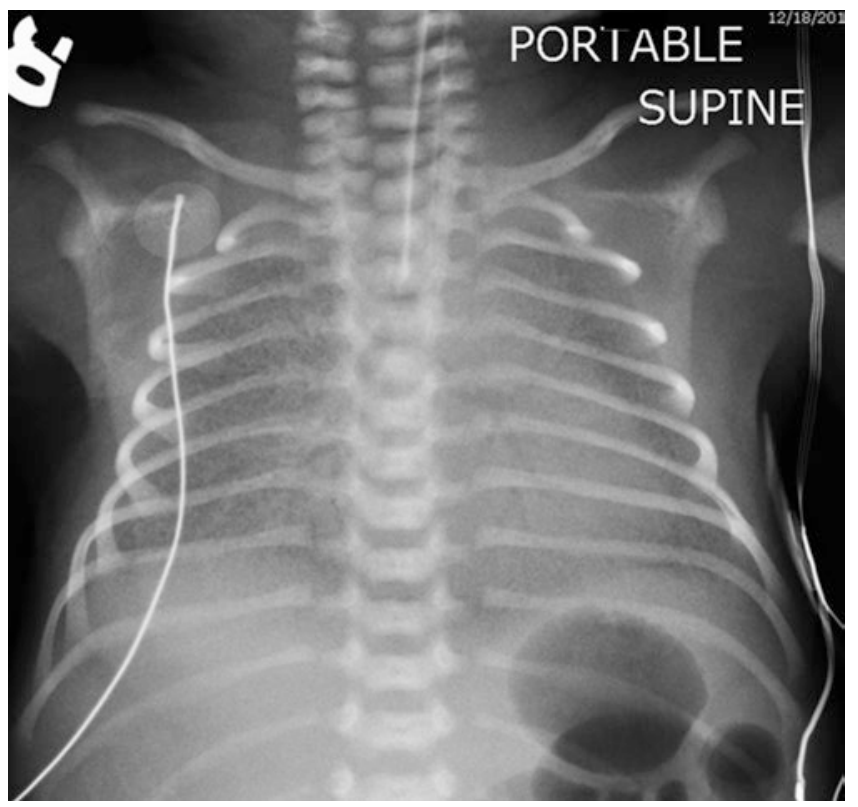

Fig. 10.1 A former 30-week gestation boy on the first day of life with surfactant deficiency of prematurity who presented with respiratory distress. Frontal chest radiograph shows diffuse symmetric hazy granular opacities in both lungs. Also noted is an endotracheal tube

support may artificially increase lung volumes or result in pneumothorax secondary to barotrauma. Inhomogeneous delivery of exogenous surfactant can result in variable aeration with areas of atelectasis distributed within persistent heterogeneous granular opacities which can mimic the appearance of meconium aspiration syndrome or neonatal pneumonia. Exogenous surfactant can also cause alveoli to hyper-expand creating bubbly lucencies that resemble the typical features of pulmonary interstitial emphysema. Additionally, antenatal maternal glucocorticoid administration can maintain patency of the ductus arteriosus resulting in pulmonary edema which manifests as diffuse pulmonary opacification with an enlarging heart radiographically.

\section{Congenital Surfactant Deficiency}

More rarely, surfactant deficiency can result from congenital genetic mutations in surfactant production and metabolism rather than solely on the basis of prematurity. Unlike surfactant disease of prematurity, congenital surfactant deficiency manifests most commonly in full-term infants who develop respiratory distress soon after birth without identifiable cause. Importantly, symptoms do not improve. Although a diverse variety of rare genetic mutations can result in surfactant deficiency, all ultimately lead to decreased oxygen exchange across the alveolar membrane.

Unlike the hyaline membranes generated in surfactant deficiency of prematurity, histologically, congenital surfactant deficiency results in diffuse alveolar hyperplasia and foamy macrophages. This histological appearance can
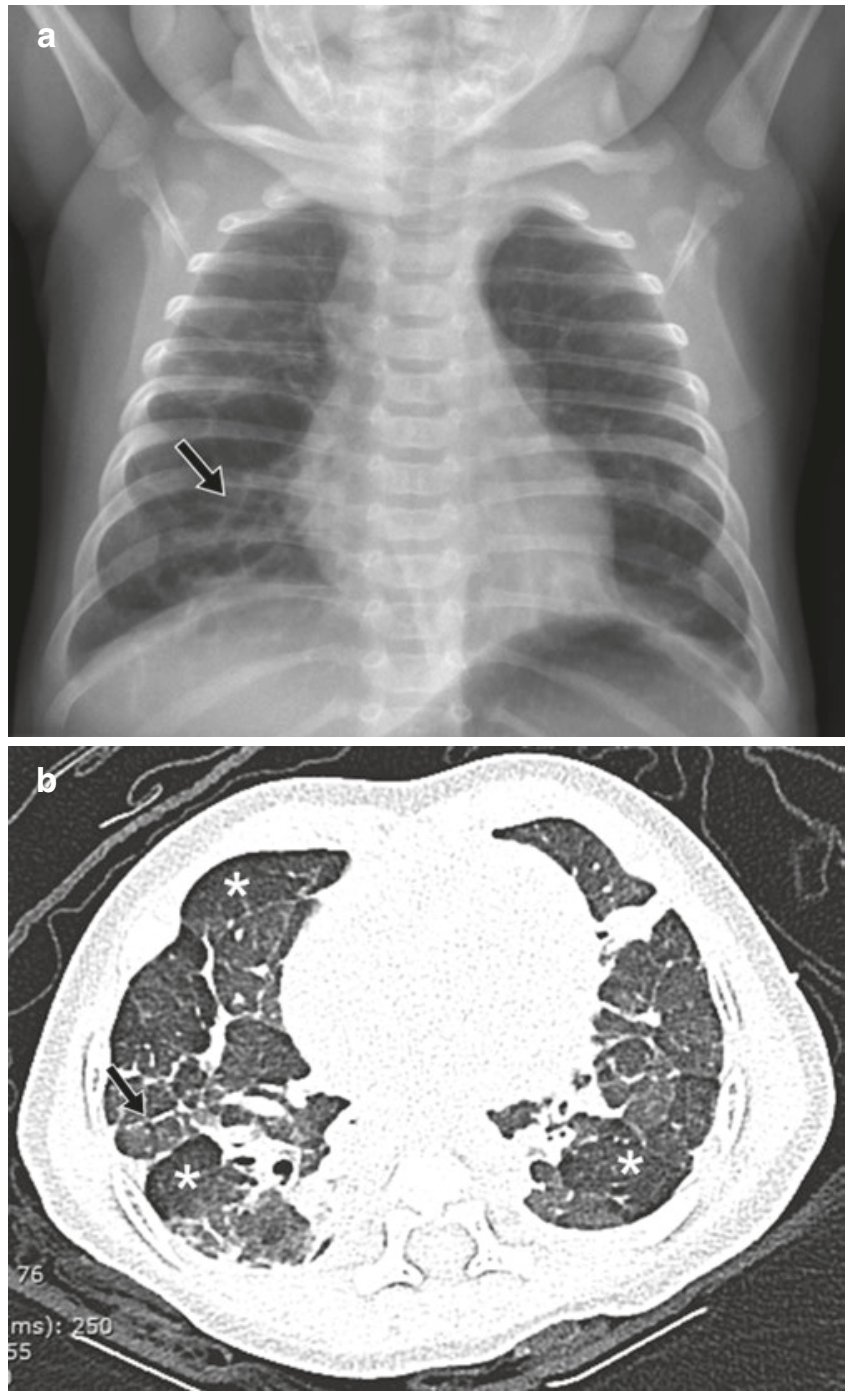

Fig. 10.2 A former 32-week gestation, now 71-day-old boy with chronic lung disease of prematurity. (a) Frontal chest radiograph shows bilateral hyperinflated lungs with coarse, reticular opacities (arrow) and architectural distortion. (b) Axial non-enhanced lung window setting CT image demonstrates coarse interstitial thickening (arrow), chronic appearing atelectasis, and areas of hyperinflation (asterisks)

sometimes mimic pulmonary alveolar proteinosis (PAP), although it is important to note that PAP is an acquired condition of children and adults rather than the result of inherent genetic mutation. Affected individuals may benefit from targeted genetic therapies for specific mutations but frequently require chronic ventilator dependence, infection prophylaxis, and long-term corticosteroids. Lung transplant may be necessary in severe cases.

Chest radiography typically demonstrates similar findings to surfactant deficiency syndrome due to prematurity including diffuse, hazy granular opacities with or without superimposed atelectasis (Fig. 10.3a). Unlike in surfactant deficiency syndrome due to prematurity, $\mathrm{CT}$ is more frequently utilized for initial evaluation and biopsy planning as pathological and 


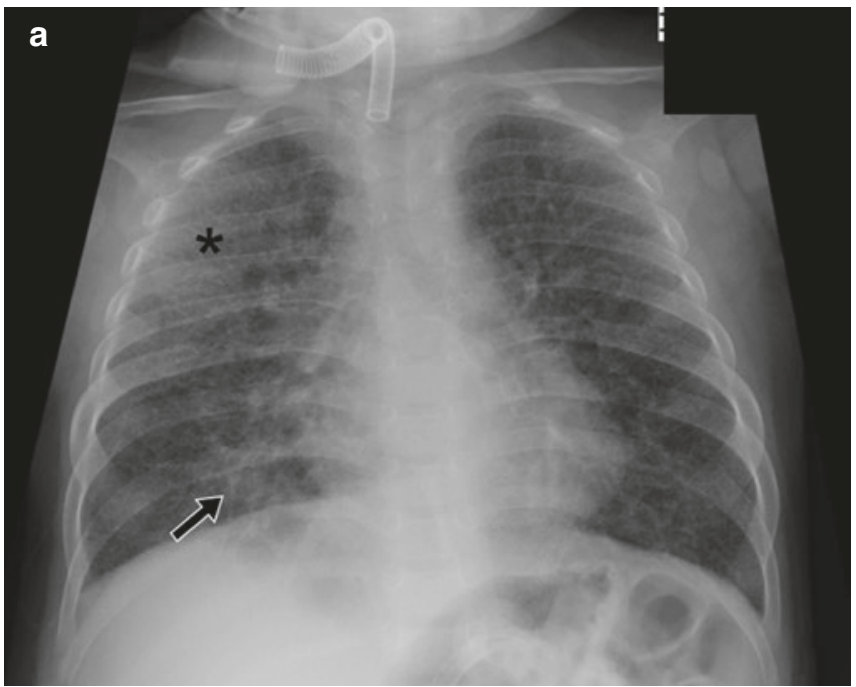

Fig. 10.3 A 13-month-old girl with congenital surfactant deficiency (ABCA3 mutation). The patient was born at term but with chronic respiratory failure since shortly after birth. Genetic testing and pathological analysis after lung biopsy confirmed the diagnosis of congenital surfactant deficiency (ABCA3 mutation). (a) Frontal chest radiograph

genetic evaluation is required in pediatric patients with congenital surfactant deficiency. On CT, diffuse ground-glass opacities are observed which eventually coalesce into thinwalled cysts, the size and number of which increase over time. Intralobular septal thickening can also result, producing an imaging pattern similar to the crazing paving observed in PAP. Superimposed consolidative airspace disease may too be present (Fig. 10.3b).

\section{Transient Tachypnea of Newborn}

Transient tachypnea of newborn (TTN) is the most common cause of respiratory distress in full-term infants. Resulting from the delayed clearance of fetal lung fluid after birth, transient tachypnea of the newborn characteristically resolves within 48-72 h without long-term sequela [10]. Typically, affected infants are normal at birth and become increasingly tachypneic over the subsequent hours developing mild to moderate respiratory distress. Fluid within the fetal lungs is normally removed after birth via the tracheobronchial tree, interstitial lymphatics, and pulmonary capillaries aided by adrenergic sodium channel activation in labor and passage through the birth canal. Incidence of TTN, therefore, increases in cesarean delivery particularly those performed before the onset of labor. Other risk factors include fetal hypotonia, postnatal sedation, delivery prior to 38 weeks gestation, male gender, low birth weight, macrosomia, and maternal gestational diabetes or asthma [11]. Supportive treatment may be required in the initial hours to days of life with affected infants recovering fully.

Radiographic findings of TTN reflect increased fluid within the interstitium including perihilar prominence and

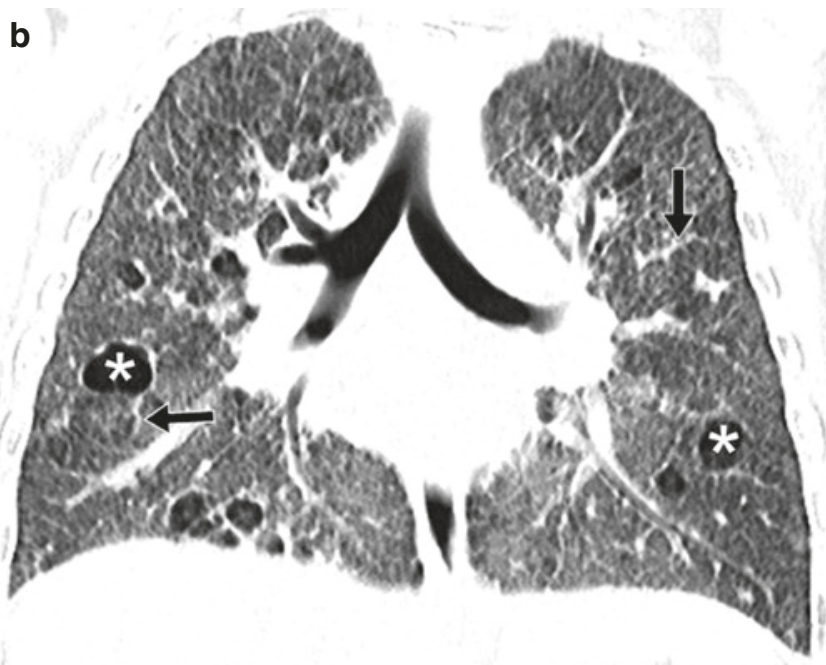

shows diffuse interstitial thickening (arrow) and ground-glass opacity (asterisk). Also noted is a tracheostomy tube. (b) Coronal non-enhanced lung window CT image demonstrates multiple small bilateral lung cysts (asterisks) in addition to diffuse, bilateral ground-glass opacity and septal thickening (arrows)

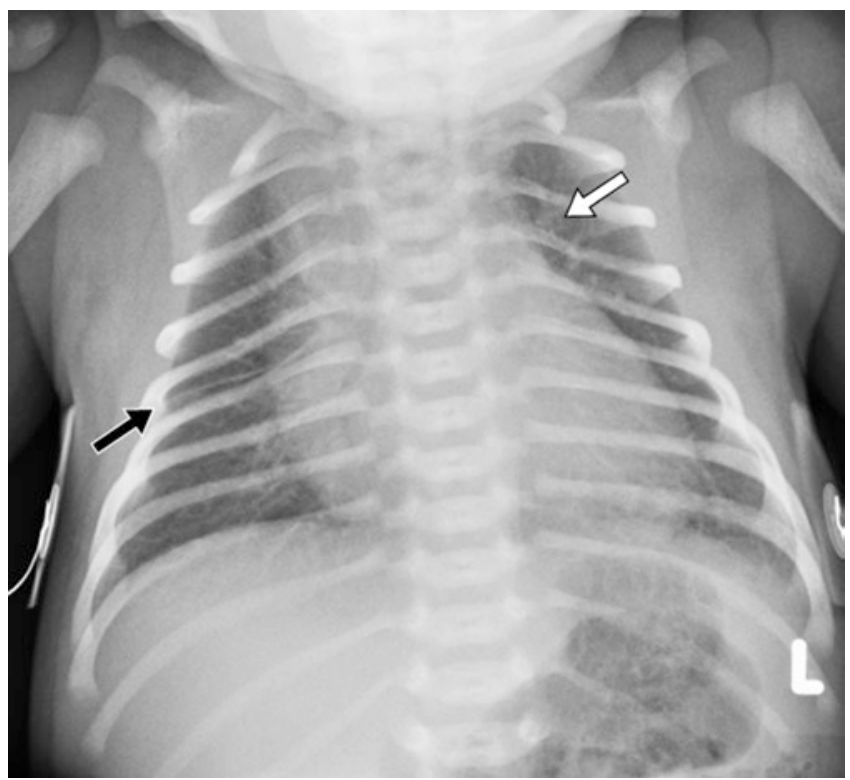

Fig. 10.4 An infant body on the first day of life born at term with transient tachypnea of newborn. The patient presented with respiratory distress. Frontal chest radiograph shows increased central interstitial markings (white arrow) and trace amount of fluid (black arrow) along the right minor fissure, suggestive of fetal lung fluid

trace fluid within the fissures [12] (Fig. 10.4). Lung volumes are typically normal to minimally increased and cardiac enlargement may be transiently present. The most specific imaging feature is improvement within 1-2 days. Infrequently, irregular bilateral opacities may be demonstrated mimicking meconium aspiration syndrome or neonatal pneumonia. Diffuse granular opacities may too be seen in 
a pattern similar to surfactant deficiency syndrome. In these cases, correlation with birth history and follow-up radiographs can aid in diagnosis.

\section{Meconium Aspiration}

Fetal inhalation of colonic material and subsequent aspiration is the most common cause of significant morbidity and mortality in full and postterm infants. Meconium is generally passed during the $24 \mathrm{~h}$ of antenatal life. Before 35 weeks gestation, however, approximately $10-15 \%$ of deliveries have meconium staining of amniotic fluid [10]. Aspiration, evidenced by the presence of meconium below the vocal cords, also increases with cesarean and postmaturity deliveries [13]. Additional risk factors include small for gestational age, intrauterine stress, and maternal factors such as hypertension, diabetes, or cardiovascular disease. The incidence of meconium aspiration has been improving in recent decades with closer fetal monitoring and improvement in obstetric practice of postterm mothers.

Aspiration of meconium produces both a mechanical obstruction secondary to plugging of small to medium airways and chemical pneumonitis which results in surfactant inactivation predisposing patients to superimposed infection. Additionally, pulmonary vasoconstriction in the setting of hypoxia may result in pulmonary hypertension. With supportive treatment, exogenous surfactant, deep suctioning, antibiotic prophylaxis, and mechanical ventilation, most patients recover within 2-3 days [10], although extracorporeal membrane oxygenation (ECMO) may be required in severe cases. Rarely, long-term complications such as pulmonary interstitial emphysema may result.

Radiographic findings of meconium aspiration vary on the severity of aspiration. Classically, lungs are hyperinflated with asymmetric, coarse, linear pulmonary opacities, frequently described as "ropelike," which radiate from the hilum. Pneumothorax should raise suspicion for meconium aspiration occurring in 10-40\% of cases [10] (Fig. 10.5). Air trapping and segmental atelectasis are frequently seen concurrent findings. Although generally not required for diagnosis, CT may be helpful in atypical cases to evaluate for other etiologies of neonatal respiratory distress or to evaluate for sequela.

\section{Neonatal Pneumonia}

Neonatal pneumonia is defined as an infection of the lower respiratory tract occurring within the first 28 days of life [10] and is the most common cause of neonatal sepsis. One of the most frequently detected underlying infectious etiologies is B. streptococcus, the transmission of which occurs during passage through an affected birth canal. However, in utero or postnatal transmission can also occur. Risk factors of neonatal pneumonia include prolonged rupture of membranes, maternal infection, mechanical ventilation, prematurity,

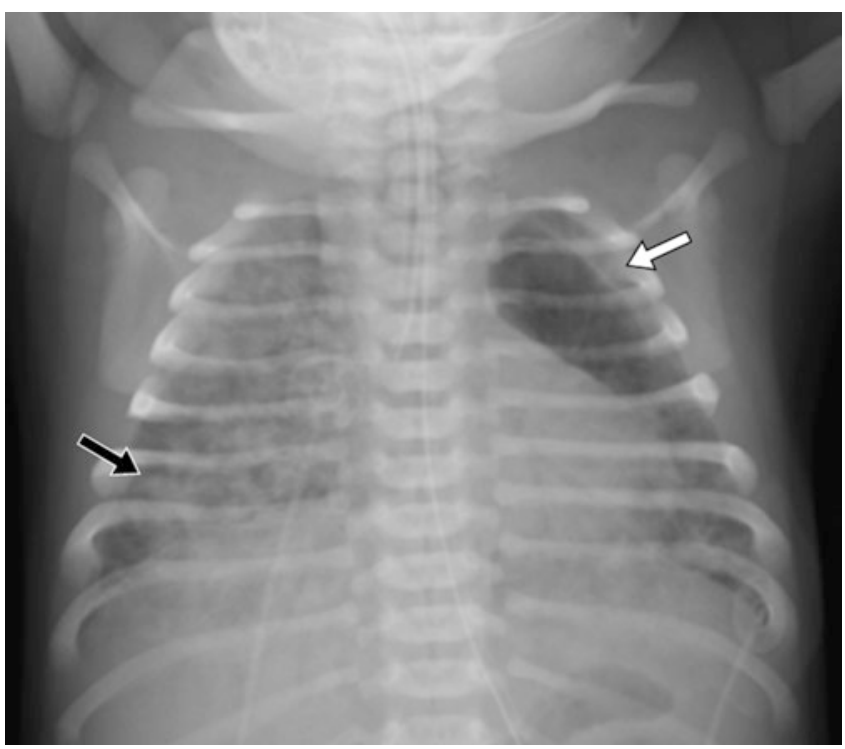

Fig. 10.5 An infant girl born at term on the first day of life who presented with respiratory distress due to meconium aspiration. She has history of meconium stained amniotic fluid at birth. Frontal chest radiograph shows hyperinflated lungs with asymmetric, coarse, linear, "ropelike" opacities (black arrow) which radiate from the hilum. An associated small left-sided pneumothorax (white arrow) is also noted

meconium aspiration syndrome, and therapeutic cooling. Although neonatal pneumonia is often associated with a significant morbidity and mortality, symptoms are frequently nonspecific including respiratory distress, labile temperature, and hypothermia. Screening mothers during the third trimester of pregnancy has decreased incidence. Empiric antibiotic therapy is the mainstay of treatment, in combination with supportive care, because blood and respiratory cultures are generally negative.

Radiographically, the imaging features of neonatal pneumonia are nonspecific, and thus a high clinical suspicion and corroborative history are required for accurate diagnosis. Diffuse and bilateral pulmonary opacities are the most frequent radiographic pattern, although focal airspace disease can also occur (Fig. 10.6). Neonatal pneumonia can also result in diffuse hazy opacities similar to surfactant deficiency syndrome or coarse irregular opacities similar to meconium aspiration syndrome. Pleural effusions are more frequently seen in neonatal pneumonia than in other causes of neonatal respiratory distress, a feature which can help differentiate these entities (Fig. 10.5a).

\subsubsection{Pulmonary Infection in Children}

\section{Round Pneumonia}

Pneumonia is the most common cause of illness in childhood and remains a significant cause of morbidity and mortality in both the developed and developing world [14]. While pediatric pneumonia can present as focal airspace opacity as is 


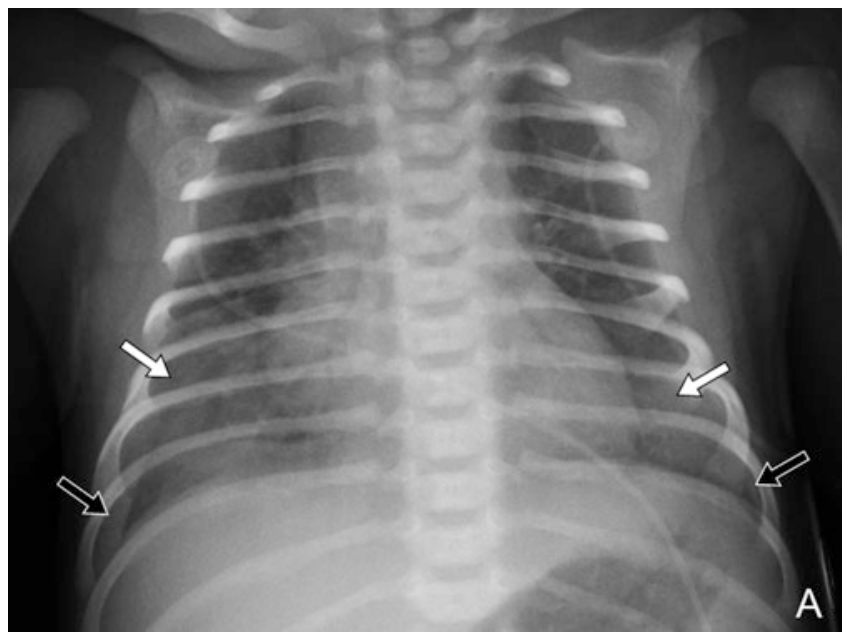

Fig. 10.6 An infant girl born at term on the third day of life with fever and respiratory distress due to neonatal pneumonia. Frontal chest radiograph shows bilateral diffuse and patchy opacities (white arrows) and trace of bilateral pleural effusions (black arrows)

classically observed in adults, round pneumonia is a distinct manifestation of lower respiratory tract infection in children less than 8 years of age [15]. Distinct differences in juvenile lung parenchyma including small alveoli, immature collateral pathways of air circulation via pores of Kohn and channels of Lambert, and closely opposed septa limit the spread of infection and round pneumonia results. Most commonly associated with Streptococcus pneumoniae, round pneumonia can also occur with Klebsiella pneumoniae and Haemophilus influenzae. Children with round pneumonia can present with more nonspecific clinical symptoms than demonstrated in adults including cough, rhinitis, vomiting, and high fever.

Radiographically, round pneumonia typically presents as a solitary, well-defined, round lesion measuring on average 3-4 cm usually located within the posterior lower lobes in contact with the adjacent pleura, hilum, or pulmonary fissure [15] (Fig. 10.7). Given the focality, size, and spherical appearance, round pneumonia may simulate a mass; although given appropriate clinical history, round pneumonia should be the primary differential consideration. Additionally, air bronchograms and satellite lesions may be present. While round pneumonia may evolve to demonstrate the more classic appearance of lobar pneumonia, with proper antibiotic treatment, 95\% resolve without complications [16].

Cross-sectional imaging is unnecessary in classic cases of round pneumonia with appropriate clinical history. CT may be warranted if there is increased clinical risk for an underlying pulmonary mass, in the absence of appropriate clinical history, or in older children as larger alveoli and better developed collateral pathways prohibit the development of round pneumonia. A round airspace opacity in these populations should prompt alternative diagnostic considerations including atypical microorganism infection, immunodeficiency, and primary or secondary malignancy. Rarely,

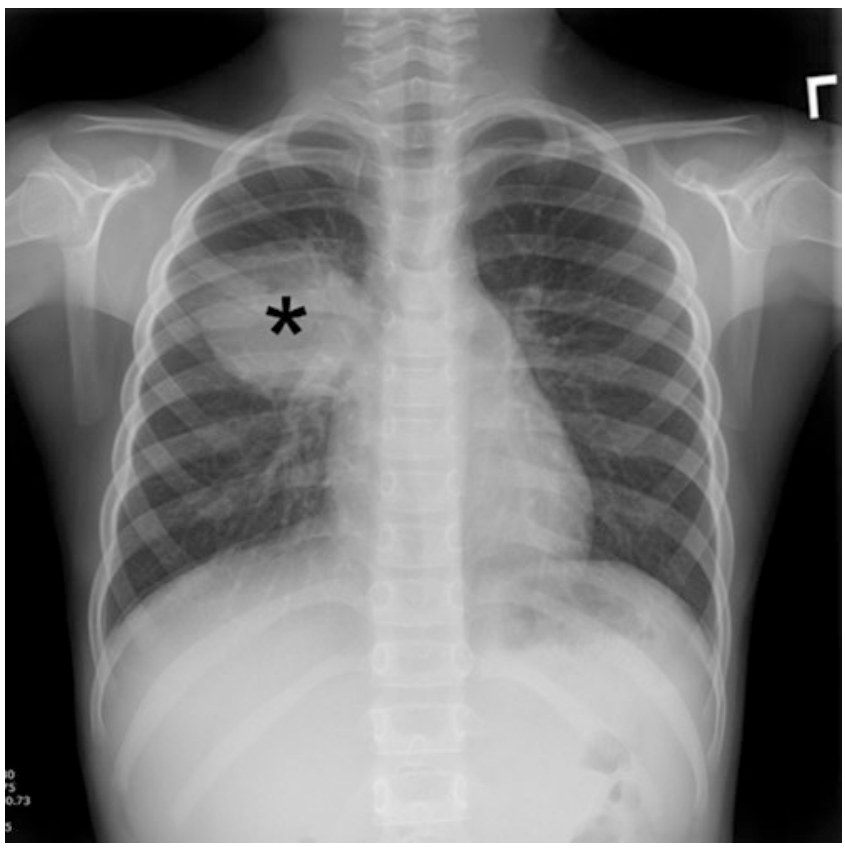

Fig. 10.7 A 5-year-old girl with cough and fever due to round pneumonia. Frontal chest radiograph shows a solitary, well-defined, and round opacity (asterisk) in the right upper to mid-lung zone adjacent to the minor fissure

complications may result in round pneumonia necessitating cross-sectional imaging evaluation by CT including cavitation, calcification, or pleural effusion. Ultrasound can be a helpful imaging modality in the evaluation of pleural and parenchymal lung abnormalities in pediatric patients because it obviates the need for sedation and radiation. In addition, ultrasound may help to distinguish lung consolidation, as in the case with round pneumonia, from a solid pulmonary mass.

\subsubsection{Pediatric Large Airway Disorders}

\subsubsection{Large Airway Neoplasms}

\section{Subglottic Hemangioma}

Subglottic hemangioma is a benign neoplasm of the subglottic airway and the most common obstructing subglottic soft tissue mass in the pediatric population. Characteristically arising along the posterior or lateral walls of the trachea, subglottic hemangioma accounts for $1.5 \%$ of congenital laryngeal malformations [17]. Although generally small, acute airway compromise can result with up to $50 \%$ mortality if untreated [17]. Symptoms typically develop at approximately 6-12 weeks of age due to postnatal lesional growth and include cough, inspiratory stridor, feeding difficulties, and hemoptysis [17]. Female, Caucasian infants are most commonly affected with up to $50 \%$ of patients also demonstrating cutaneous hemangiomas. Additional risk factors include low birth weight, prematurity, and multiple gestations [18]. 

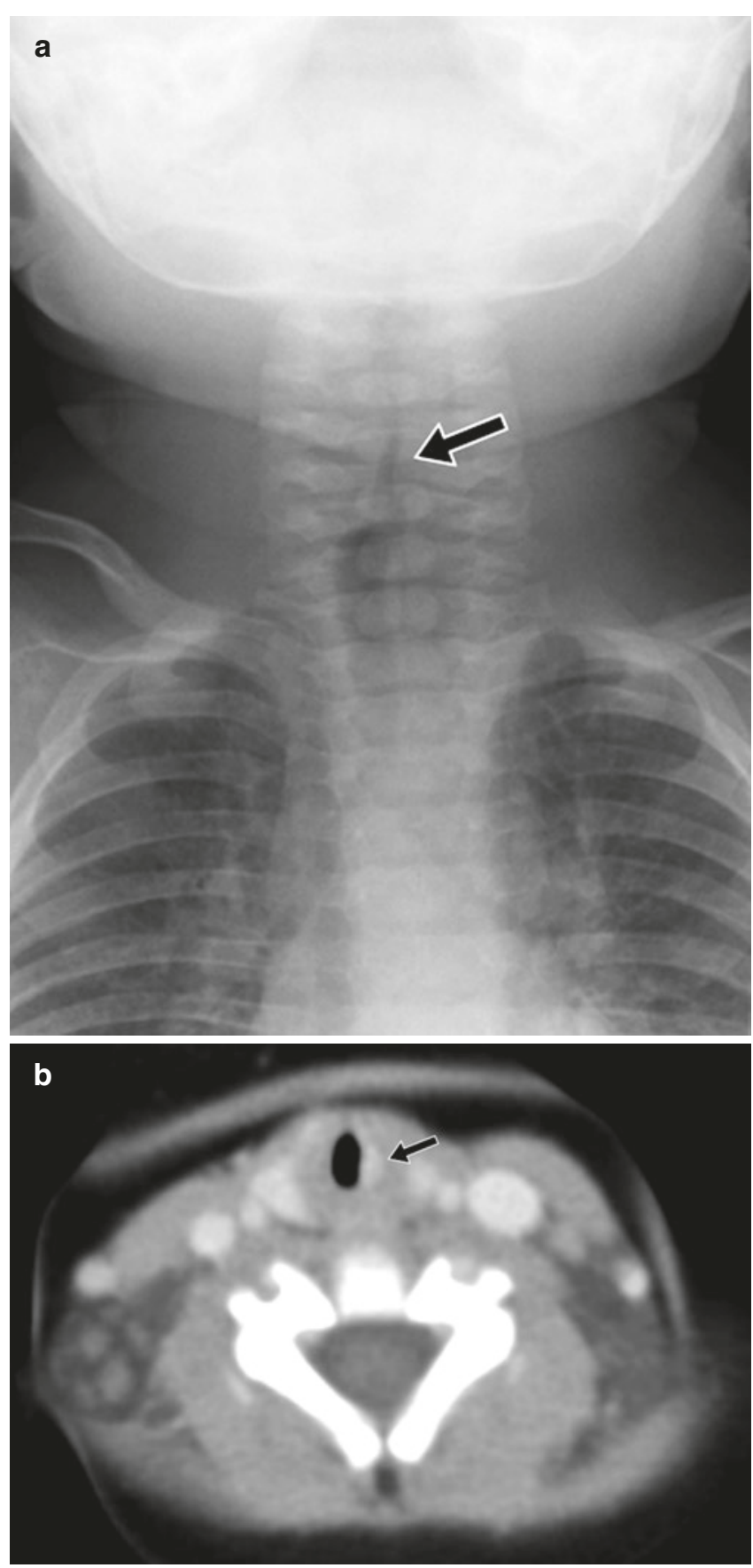

Fig. 10.8 A 3-month-old boy with subglottic hemangioma who presented with stridor. (a) Frontal soft tissue neck radiograph shows eccentric narrowing (arrow) of the left subglottic trachea. (b) Axial enhanced soft tissue window setting CT at the level of the subglottic trachea demonstrates a well-circumscribed, avidly enhancing soft tissue lesion (arrow) at the lateral aspect of the left subglottic trachea

The classic radiographic appearance of subglottic hemangioma is nonspecific, eccentric narrowing of the subglottic trachea on frontal views [19]. Such asymmetric appearance can help differentiate from other causes of subglottic tracheal narrowing such as croup (Fig. 10.8a). Following radiography, cross-sectional evaluation with CT or MRI is routinely performed for pretreatment planning with either modality typi- cally demonstrates an avidly enhancing lesion at the posterolateral aspect of the subglottic trachea with soft tissue characteristics (Fig. 10.8b) [3, 20, 21].

Novel treatment techniques such as laser ablation, propranolol injection, or sclerotherapy have become the standard of care in the treatment of subglottic hemangioma, thereby reducing the morbidity associated with surgical excision.

\section{Carcinoid Tumor}

Carcinoid tumor is the primary neuroendocrine tumor of the lung. Although rare and less common than carcinoid tumor of the gastrointestinal tract, it is the most common primary lung tumor in the pediatric population with a mean age of presentation of 14 years [22]. A centrally located carcinoid tumor within a large airway is more likely to result in symptoms in comparison to a carcinoid tumor located more peripherally within the lung parenchyma. Affected children typically present with cough, shortness of breath, hemoptysis, or, most commonly, recurrent pneumonias. Histologically, carcinoid tumor can be classified as typical or atypical with typical lesions demonstrating fewer mitoses per high power field, lower likelihood of necrosis, and less malignant potential.

Radiographically, carcinoid tumor can present as a wellcircumscribed, round, or ovoid lesion. However, if central in location, it may be obscured by hilar structures and only secondary signs observed such as atelectasis or pneumonia (Fig. 10.9a). The parallel sign has been described in the literature to describe the orientation of a non-spherical carcinoid tumor along axis of the nearest major bronchus or pulmonary artery. Cross-sectional imaging with CT or MRI is important for presurgical planning and typically demonstrates a well-defined or lobulated lesion with soft tissue attenuation characteristics and robust post-contrast enhancement (Fig. 10.9b) [23, 24]. Associated calcification may be demonstrated more commonly with central lesions versus those in the periphery. ${ }^{111}$ In-labeled pentetreotide (Octreoscan) is suggested by the Pediatric Carcinoid Tumor Clinical Guidelines to assess for sites of metastatic disease.

Treatment is primarily surgical with low recurrence rates observed with segmentectomy or wedge resection. Chemotherapy or somatostatin-based therapies are reserved for unresectable cases.

\subsubsection{Nonneoplastic Disorders of the Large Airway}

\section{Tracheomalacia}

Tracheomalacia is defined as dynamic and disproportionate collapse of the tracheal lumen with expiration [25]. It results from weakening of the supporting cartilage, softening of the airway walls, or hypotonia of the supporting muscles [26]. Affecting both premature and term infants, tracheomalacia 

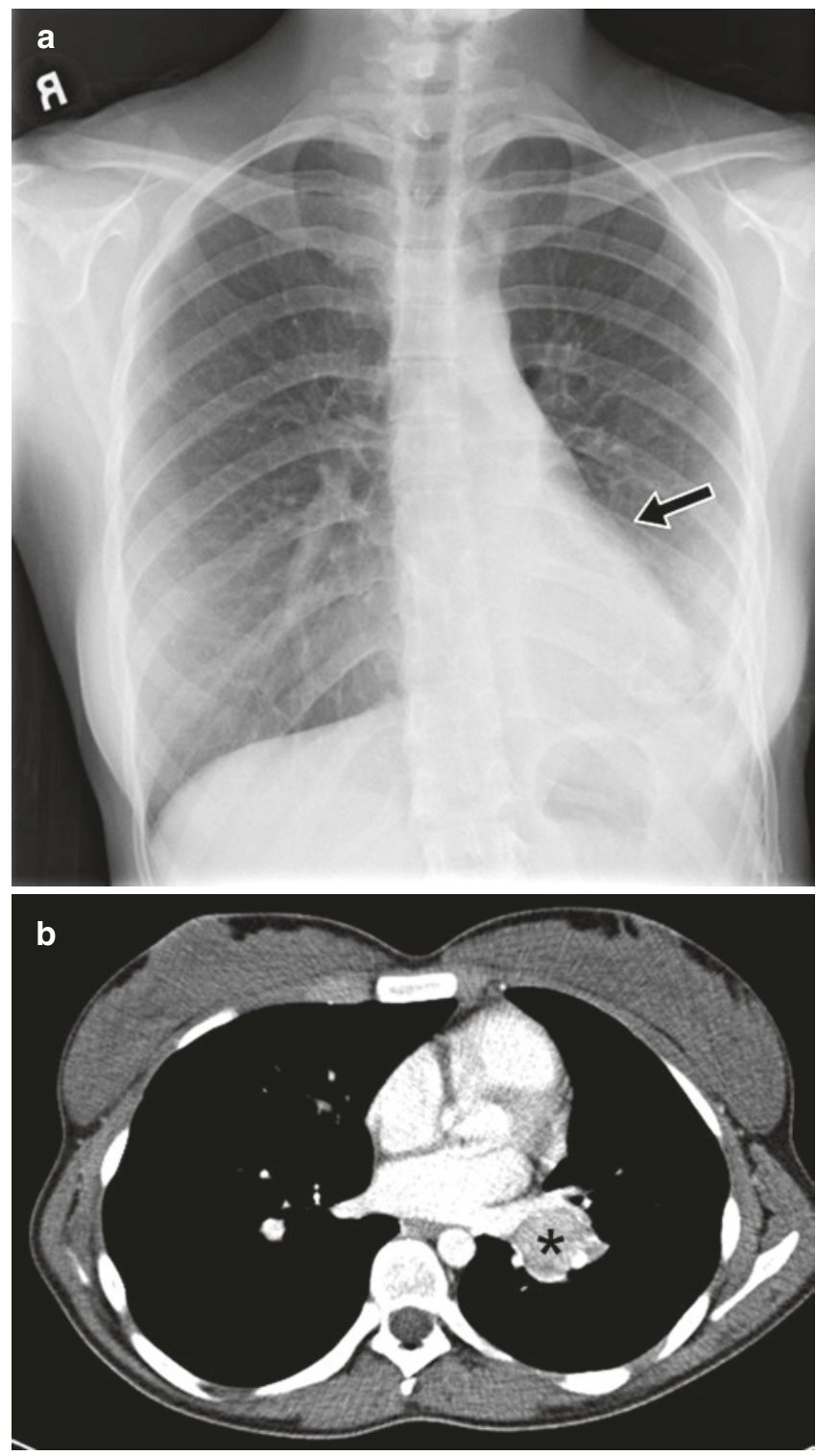

Fig. 10.9 A 16-year-old girl with endobronchial carcinoid who presented with cough and shortness of breath. The patient underwent surgical excision of the endobronchial mass in the left lower lobe bronchus and histological analysis confirmed the diagnosis of low-grade carcinoid tumor. (a) Frontal chest radiograph shows an opacity with volume loss in the left lower lobe (arrow) due to left lower lobe collapse. (b) Axial enhanced soft tissue window setting CT image, at the level of the left atrium, demonstrates a well-circumscribed, heterogeneously enhancing soft tissue mass (asterisk) near the left hilum

may be due to primary, congenital causes such as prematurity, tracheoesophageal fistula, or congenital cartilage disorder or may be acquired secondarily as a result of intubation, infection, surgery, extrinsic compression from vascular anomaly, cardiovascular defects, or neurologic causes. Typical symptoms include cough, recurrent lower airway infection due to decreased clearance, dyspnea, recurrent wheeze, or stridor with symptoms frequently worsening with activity or agitation.

Fluoroscopic measurement of the tracheal diameter has a limited role in the evaluation of tracheomalacia with a specificity of $96-100 \%$ but a sensitivity of only $23.8-62 \%$ because the trachea of a normal infant may collapse up to $50 \%$ with forceful crying [27]. A barium esophagram may help to evaluate causes of acquired tracheomalacia such as due to tracheoesophageal fistula or extrinsic compression due to vascular ring but with limited anatomic detail. In intubated infants using breath-hold technique as well as older children who can follow breathing instructions, CT with paired inspiratory and expiratory series can be used to evaluate precise localization, extent, and severity of tracheomalacia [28]. In recent years, with the availability of higher row multidetector CT (MDCT), real-time and dynamic 3D and 4D imaging of the large airway can be obtained even in infants and young children who cannot follow breathing instructions obviating the need for intubation (Fig. 10.10a-d). Additionally, CT allows for the identification of underlying causes as well as associated anomalies such as air trapping.

Cases of primary tracheomalacia due to prematurity and intrinsic weakness of the supporting cartilage may require only supportive care during the first 1-2 years of life after which normal stiffening of the cartilage occurs and the condition self-resolves [27]. In severe cases, aggressive treatment may be required including continuous positive airway pressure, tracheostomy, airway stenting, or surgical repair with pexy procedures. Recently, customized, bioresorbable 3D-printed splints have been used in cases of severe tracheomalacia with preliminary success in both pediatric and adult patients although larger and longer studies are necessary to evaluate long-term outcomes and complications [29].

\section{Foreign Body Aspiration}

Because infants and young children aged 6 months to 3 years lack molar teeth, have uncoordinated swallowing mechanisms, and, most importantly, engage with their surroundings by placing objects in their mouths, they are prone to foreign body aspiration [30]. Aspirate objects, most commonly peanuts, lodge with the highest frequency within the right main stem bronchus due to its larger size and more vertical alignment as compared with the left.

Chest radiography is the first-line imaging study in cases of suspected foreign body aspiration because it is readily available, low in cost, and associated with minimal radiation exposure [31]. As only $10 \%$ of aspirated foreign bodies are radiopaque [32], however, indirect signs of aspiration including air trapping, focal airspace disease, pleural effusion, mediastinal shift, pneumothorax, or subcutaneous emphysema are important imaging surrogates. Unilateral hyperinflation [33] is the most commonly identified indirect sign of aspiration [34] (Fig. 10.11a). This finding may be further evaluated with bilateral decubitus radiographs to assess for air trapping with the side of foreign body aspiration failing to deflate in the dependent position (Fig. 10.11b and c). Lateral 

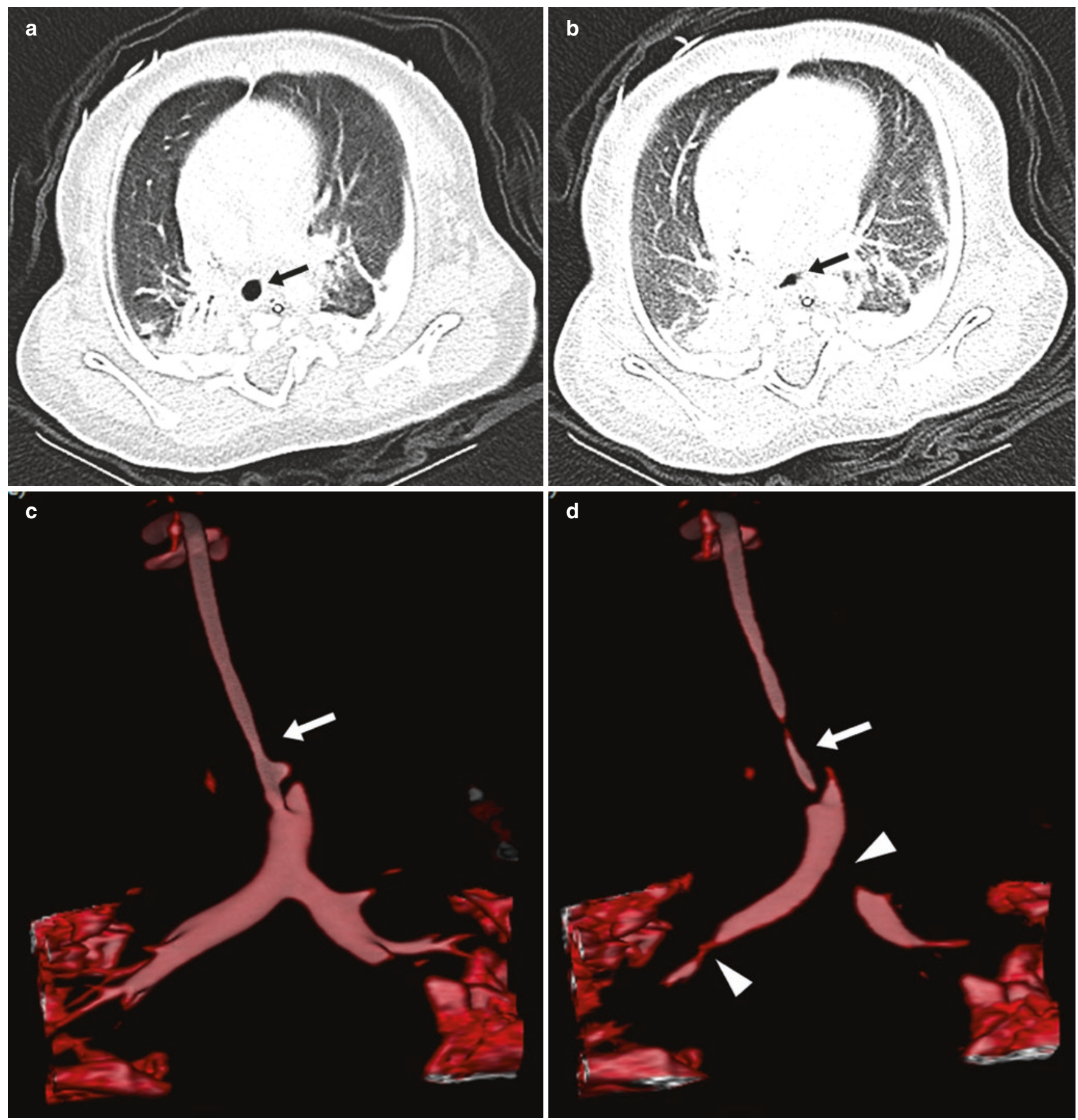

Fig. 10.10 A 3-month-old girl with tracheobronchomalacia who presented with recurrent wheezing and stridor. Subsequently obtained bronchoscopy confirmed the diagnosis of tracheobronchomalacia. (a) Axial non-enhanced lung window setting CT image, at the level of the mid-thoracic trachea, obtained at end-inspiration shows patent trachea (arrow). (b) Axial non-enhanced lung window setting CT image, at the level of the mid-thoracic trachea, obtained at end-expiration demonstrates focal collapse of the trachea (arrow) with greater than $50 \%$ decrease in cross-sectional area consistent with tracheomalacia. (c) 3D

volume-rendered CT image of the large airways obtained at endinspiration shows patent trachea (arrow). Patent bilateral main stem bronchi are also seen. (d) 3D volume-rendered CT image of the large airways obtained at end-expiration demonstrates focal marked collapse of the trachea (arrow) consistent with tracheomalacia. Also noted is similar focal marked collapse (arrowheads) of the distal right main stem bronchus and proximal left main stem bronchus, consistent with bilateral bronchomalacia 

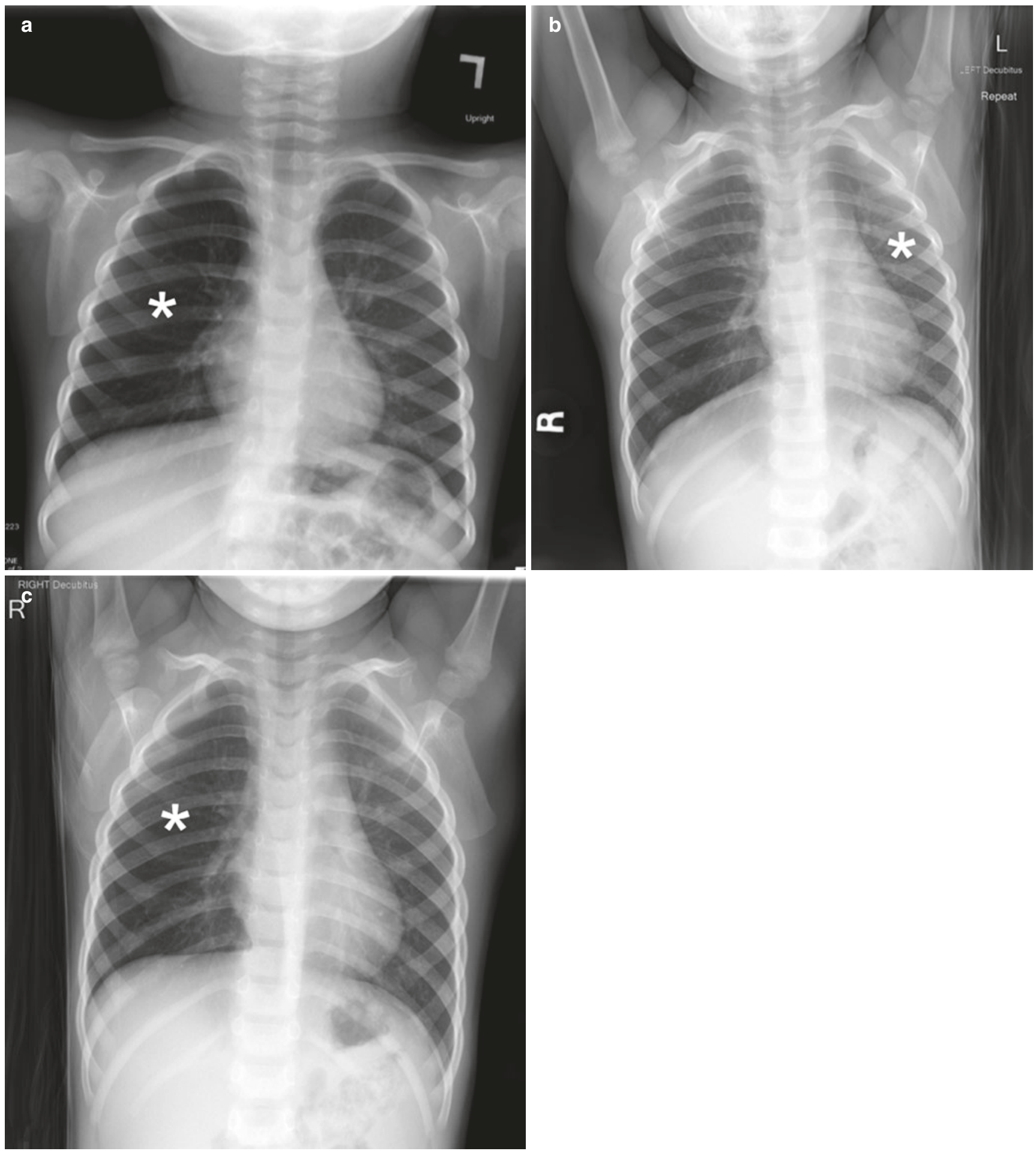

Fig. 10.11 A 30-month-old boy with an endobronchial non-radiopaque foreign body aspiration who presented with acute onset of wheezing and respiratory distress. (a) Frontal chest radiograph shows asymmetric hyperinflation of the right lung (asterisk) as compared with the normally aerated left lung. (b) Left lateral decubitus radiograph demonstrates expected deflation of the left lung (asterisk) in the dependent portion. (c) Right lateral decubitus radiograph shows persistent relative hyperinflation representing air trapping of the right lung (asterisk) despite dependent positioning. Radiographic findings suspicious for aspirated non-radiopaque foreign body in the right main stem bronchus. Subsequently obtained bronchoscopy demonstrated a nearly obstructive foreign body (likely almond) located within the right main stem bronchus 
decubitus radiography, however, have only $68-74 \%$ sensitivity and 45-67\% specificity [35]. Furthermore, it has been shown to increase false-positive rates without increasing the rate of true-positive identification. As such, a negative chest radiography in the setting of high clinical suspicion should prompt further evaluation with CT.

CT has an accuracy close to $100 \%$ for the detection of aspirated foreign body [35] able to identify both an endoluminal mass as well as secondary signs of aspiration. Because of the increased radiation exposure of $\mathrm{CT}$ as compared with radiography, study benefits must be weighed. 3D virtual bronchoscopy reconstructions can be utilized to assess foreign body aspiration and may be used for preprocedural planning. Additionally, following bronchoscopic removal, CT can be used to assess for residual aspirated foreign body [36].

\subsubsection{Pediatric Mediastinal Disorders}

\subsubsection{Vascular Lesions}

Vascular rings are congenital abnormalities of the aortic arch which result from abnormal development of the aortic arches formed during the fourth and fifth weeks of embryonic development. Depending upon the location of failed arch regression, various anomalous anatomic variants may result which encircle the trachea and esophagus to various degrees. While some remain asymptomatic and may be incidental findings later in life, others, namely, double aortic arch and right aortic arch with aberrant left subclavian artery, result in symptoms earlier in life and thus frequently present to clinical attention in early infancy. Abnormalities of aortic arch development may be associated with additional congenital cardiac anomalies, as is frequently the case with right aortic arch with mirror image branching, while others are isolated findings without additional congenital associations.

\section{Double Aortic Arch}

A double aortic arch is caused by persistence of the both the right and left embryonic arches which arise from the ascending aorta. Because a complete vascular ring encircling the trachea and esophagus is formed, affected pediatric patients generally develop symptoms early in life including wheezing, stridor, cyanosis, and dysphagia frequently exacerbated by activity or crying. In a double aortic arch, one arch is usually dominant with the descending aorta typically located on the side contralateral to the dominant arch, most commonly manifesting as a dominant right arch with left-sided descending aorta. A double aortic arch is rarely associated with additional congenital heart abnormalities.

Radiographically, a double aortic arch demonstrates bilateral aortic knobs on the frontal projection with dis- placement of the tracheal air column away from midline and indentation upon the right lateral tracheal border (Fig. 10.12a). On the lateral projection, the retrotracheal aorta may result in anterior displacement of the trachea. On barium esophagram, there may be bilateral indentations on the esophagus on frontal views and posterior indentation on the lateral view.

Whereas the presence of a double aortic arch may be inferred radiographically or fluoroscopically, cross-sectional imaging with CT or MRI has improved sensitivity and results in superior anatomic detail allowing for elucidation of relational anatomy to other mediastinal structures [37]. Characteristic of a double aortic arch is a symmetric vascular branching pattern demonstrated on single axial image at the level of the thoracic inlet consisting of the origins of the common carotid and subclavian arteries bilaterally, so-called the "four-vessel" sign (Fig. 10.12b). Cross-sectional imaging is also useful for planning of surgical approach with reformatted images offering important insight into arch size, position, and dominance (Fig. 10.12c).

The current management of double aortic arch involves surgical ligation of the nondominant arch usually via an ipsilateral thoracotomy.

\section{Right Aortic Arch}

In normal development, a left aortic arch forms from the involution of the right fourth arch and dorsal aorta. When there is involution of the left fourth arch and dorsal aorta, which occurs in $0.1 \%$ of the population [38], a right aortic arch results. Right aortic arch is the second most common aortic arch variant after double aortic arch. There are two main types of right aortic arch: right aortic arch with an aberrant subclavian artery and right aortic arch with mirror imaging branching. A right arch may be associated with an aberrant left subclavian artery arising from or independent to a Kommerell diverticulum occurring between the left common carotid and left subclavian arteries. A right aortic arch may also be associated with mirror imaging branching where the embryonic arch is interrupted just distal to the left ductus arteriosus. In addition, although the right aortic arch typically descends within the right aspect of the thoracic cavity, rarely the arch can cross to the midline posterior to the esophagus with the descending aorta located on the left as occurs in the case of right circumflex aorta. Such vascular anatomy creates a vascular ring which is completed by a left ductus or ligamentum arteriosum. Although a right aortic arch with an aberrant left subclavian artery is infrequently associated with additional congenital heart anomalies, a right arch with mirror image branching is highly associated with accompanying congenital heart anomalies (90-98\%) most commonly Tetralogy of Fallot [39]. 

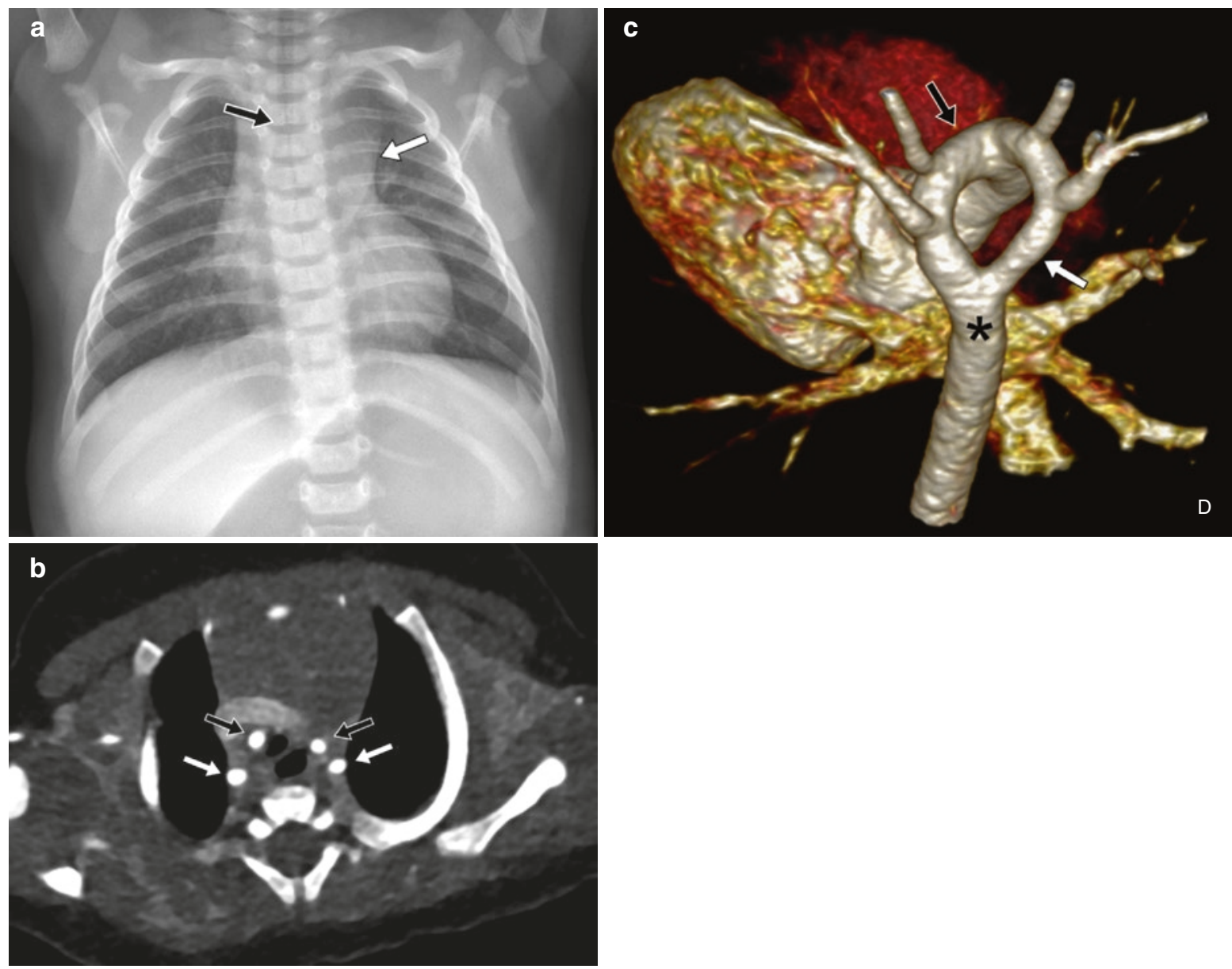

Fig. 10.12 A 74-day-old girl with double aortic arch who presented with wheezing and feeding difficulty. (a) Frontal chest radiograph shows minimal leftward deviation (black arrow) of thoracic trachea with indentation along the right lateral tracheal wall. There is also prominence (white arrow) of the superior mediastinum. (b) Axial enhanced soft tissue window setting CT image, at the level of thoracic inlet, demonstrates a symmetric vascular branching pattern comprised

Chest radiography characteristically demonstrates a right-sided aortic arch with variable positioning of the descending aorta (Fig. 10.13a). In cases of right aortic arch with mirror image branching, findings associated to the accompanying congenital heart disease may be present such as situs inversus or cardiac enlargement. As in cases of double aortic arch, cross-sectional evaluation with CT or MRI again provides superior anatomic resolution and allows for clarification of mediastinal relationships (Fig. 10.13b and c).

Management of right aortic arch depends on associated anomalies and symptomatology. An asymptomatic right arch with aberrant left subclavian artery requires no treatment. However, surgical ligation is required in symptomatic pediatric patients with right aortic arch constituting a vascular ring. Repair of underlying cardiac anomalies may be also needed in pediatric patients with right aortic arch with mirror imaging branching.

of the origins of the bilateral common carotid arteries (black arrows) and bilateral subclavian arteries (white arrows), the so-called the fourvessel sign. (c) Posterior view of 3D volume-rendered CT image shows double aortic arch anatomy including the left (black arrow) and right (white arrow) aortic arches which comprise a complete vascular ring. Right-sided descending aorta (asterisk) is also seen

\section{Pulmonary Artery Sling}

A pulmonary artery sling results from failure of development of the left sixth aortic arch. As a result, there is anomalous origin of the left pulmonary artery from the right pulmonary artery with the left pulmonary artery then passing between the trachea and esophagus as it courses toward the left hilum. Consequently, compression of the trachea and right main stem bronchus may result with secondary development of tracheobronchomalacia or stenosis. A pulmonary artery sling is classified according to the associated airway abnormalities. Type I results in compression of the trachea and right main stem bronchus with normal airway branching. Type II often results in long segment tracheobronchial stenosis often with associated abnormal airway branching with development of a T-shaped carina.

Radiographically, a pulmonary sling may manifest as unilateral hyperinflation, low position of the left hilum, and anterior displacement of the trachea with posterior indenta- 


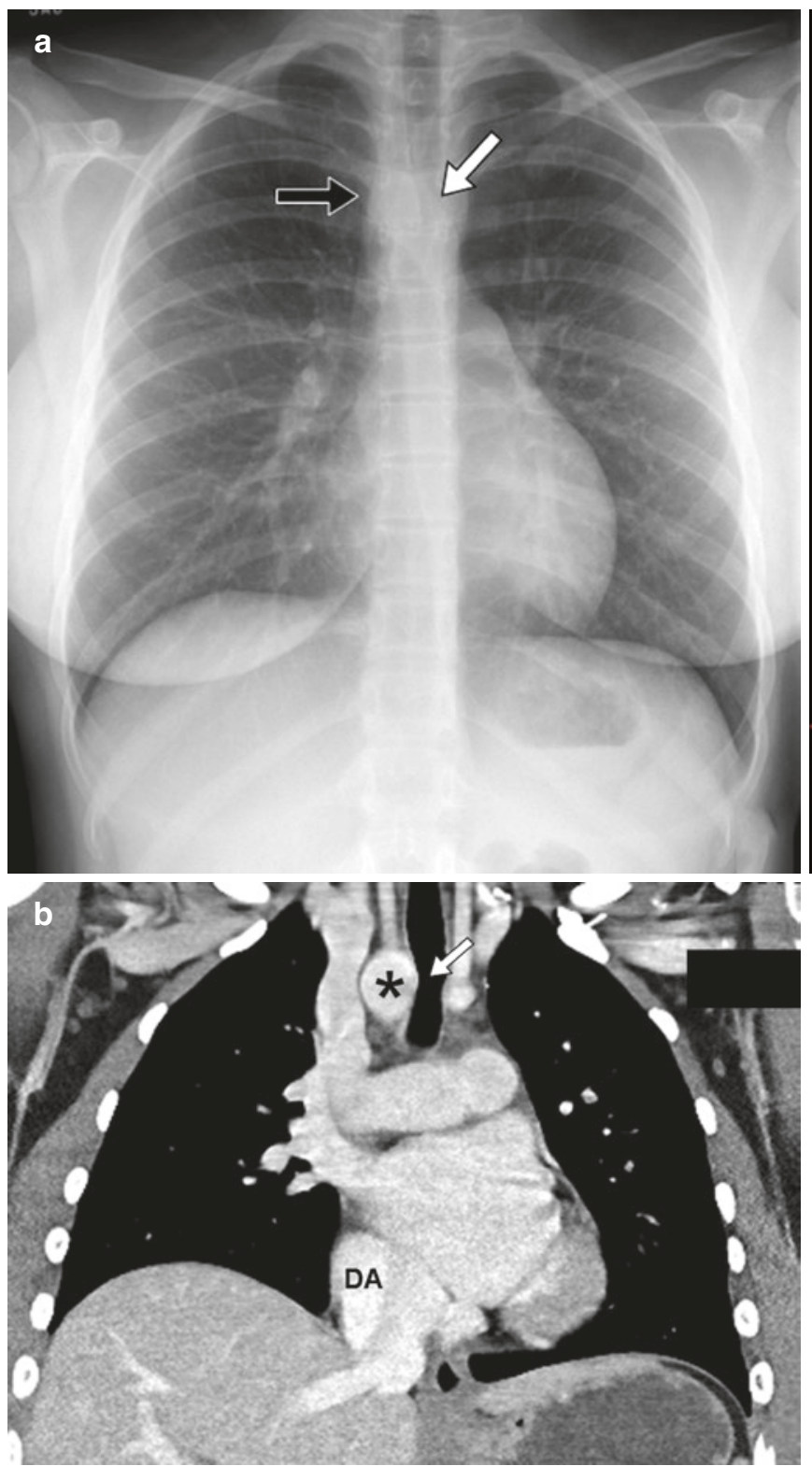

Fig. 10.13 A 15-year-old girl with a right aortic arch who presented with wheezing. (a) Frontal chest radiograph shows right-sided aortic knob (black arrow) which indents upon and leftward deviation (white arrow) of the thoracic trachea. (b) Coronal enhanced soft tissue window setting CT demonstrates a right-sided aortic arch (asterisk) and descend-

tion. Barium esophagram classically demonstrates anterior indentation on the esophagus. Cross-sectional imaging again better defines the vascular and airway anatomy while aiding in the exclusion of right pulmonary agenesis as a differential consideration. Additionally, CT can aid in the evaluation of secondary tracheobronchomalacia and stenosis. Crosssectional imaging is also helpful for presurgical evaluation because treatment usually involves median sternotomy, cardiopulmonary bypass, reimplantation of the anomalous left pulmonary artery, and repair of large airway anomaly or abnormality if present (Fig. 10.14) [40].

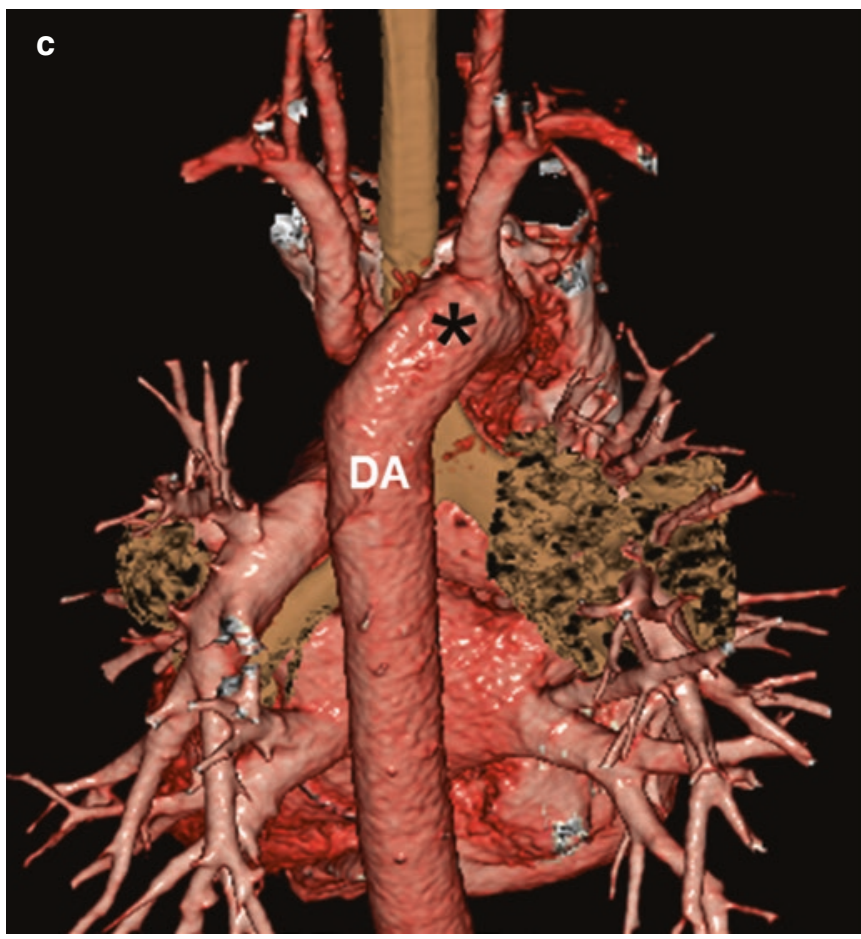

ing aorta (DA). Compression (arrow) of the trachea due to right aortic arch is also seen. (c) Posterior view of 3D volume-rendered CT image demonstrates right aortic arch anatomy with right-sided descending aortic arch (asterisk) and descending aorta (DA)

\subsubsection{Nonvascular Lesions}

\section{Foregut Duplication Cyst}

Foregut duplication cyst is the most common primary mediastinal mass and comprises $11 \%$ of all mediastinal masses [41]. It is believed to arise from disturbances in embryologic differentiation of the primitive gut which typically develops into the pharynx, respiratory tract, esophagus, stomach, and the proximal duodenum [42]. In general, symptoms relate to the level of cyst, mass effect by the cyst on adjacent structures, and the specific cells involved in the cyst. 


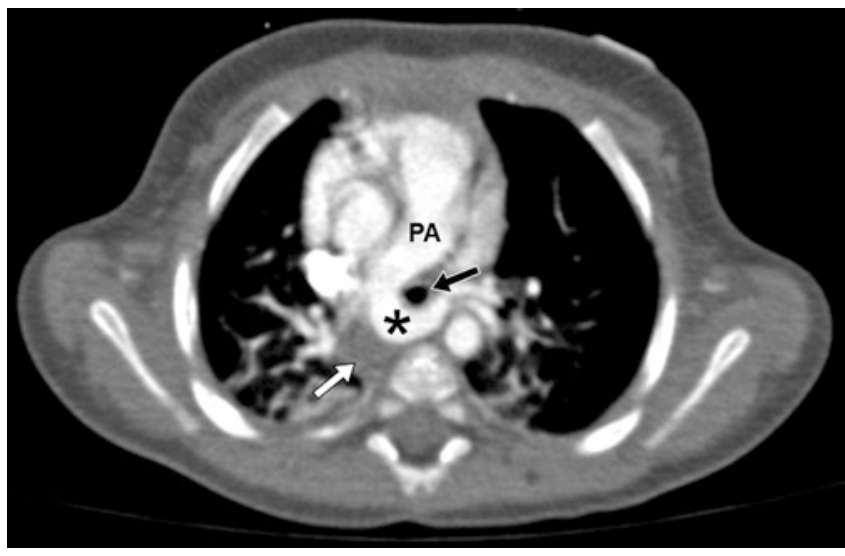

Fig. 10.14 A 2-month-old girl with pulmonary artery sling who presented with respiratory distress. Axial enhanced soft tissue window setting CT image demonstrates anomalous origin of the left pulmonary artery (asterisk) from the main pulmonary artery (PA). The left anomalous pulmonary artery (asterisk) passes between the trachea (black arrow) and esophagus (white arrow) as it courses toward the left hilum

Foregut duplication cyst can be broadly classified into three types which include bronchogenic cyst, esophageal duplication cyst, and neurenteric cyst. Bronchogenic cyst arises from abnormal lung budding during ventral foregut division and can occur anywhere along the tracheoesophageal tree, most commonly in the subcarinal or paratracheal regions. Although usually asymptomatic, if large, bronchogenic cyst can result in dyspnea from secondary compression of an adjacent airway. Esophageal duplication cyst is the result of abnormal division of the posterior embryonic foregut. It is most commonly associated with dysphagia as the duplication cyst may occur anywhere along the esophagus, though most arise in the upper one third. A failure of complete separation of the gastrointestinal tract from the primitive neural crest can result in a neurenteric cyst which is one variant of split notochord syndrome. Usually occurring in the posterior mediastinum, neurenteric cyst may communicate with the spinal cord resulting in pain as well as an associated cleft within the adjacent vertebral body.

Radiographically, foregut duplication cysts of all subtypes manifest as smooth, oval, or round middle or posterior mediastinal masses (Fig. 10.15a) requiring further crosssectional imaging with CT or MRI evaluation prior to surgical removal [41, 43]. Although neurenteric cyst may have an associated vertebral body anomaly, other imaging features are less specific with a well-marginated cystic lesion most commonly identified. Uniform fluid attenuation or homogeneous T2 hyperintensity is demonstrated in up to one half of lesions (Fig. 10.15b). Heterogeneity, however, can result in the setting of prior hemorrhage, infection, or increased internal protein although most lesions still retain intrinsic T2 hyperintensity. After administration of contrast, irregular border and peripheral contrast enhancement may also be identified in the setting of prior or superimposed infection (Fig. 10.15c).

The current management of symptomatic pediatric patients with foregut duplication cysts is surgical resection.

\section{Neuroblastoma}

Thoracic neurogenic tumors may arise from any component of the nervous system within the chest. They are the most common posterior mediastinal masses in children, accounting for $90 \%$ of posterior mediastinal masses [41]. Mediastinal location of neuroblastoma is associated with more benign and mature subtype of neuroblastoma as compared with intraabdominal location. Thoracic neurogenic tumors can be separated into two main categories based upon the origin of their precursor cell from either an autonomic nerve ganglia or the nerve sheath. Lesions of autonomic nerve ganglia origin are more commonly encountered in pediatric patients with differential consideration ranging from the benign ganglioneuroma to the malignant neuroblastoma with ganglioneuroblastoma having histological features of each and being of transitional malignant potential. Although imaging alone is not possible to differentiate these tumor types, imaging plays an important role in identifying and characterizing the mass and its extent.

Neuroblastoma is the most common posterior extracranial solid malignancy of childhood and most frequently occurs in patients less than 3 years of age. Depending on primary tumor location, presenting clinical symptoms may include Horner syndrome or heterochromia if the primary tumor is cervical or apical in location, paraplegia or loss of bladder/bowel function if there is spinal cord compression, or opsoclonus-myoclonus syndrome if circulating autoantibodies are present. In addition, associated nonspecific symptoms may be present including fever, irritability, weight loss, anemia, and pain. Levels of catecholamines or neurotransmitters, which are detected by blood or urine tests, are typically elevated in pediatric patients with neuroblastoma.

Classically included in the radiographic differential of a posterior mediastinal lesion, neuroblastomas frequently demonstrate associated calcification $(50 \%)$ and may cause secondary osseous findings including rib erosion or enlargement of the neuroforamen [44] (Fig. 10.16a and b). Neuroblastoma usually lacks a well-defined capsule and may invade adjacent mediastinal structures or metastasize. Cross-sectional imaging with CT or MRI provides crucial staging information including location, lesion size, and the presence of metastatic disease (Fig. 10.16c). MRI is especially helpful in the classification of extradural disease due to the intrinsic high lipid and water content of these tumors which results in both $\mathrm{T} 1$ and $\mathrm{T} 2$ hyperintensity. MRI, in comparison to CT, can also better demonstrate the extension of thoracic neuroblastoma into the adjacent neural foramen. 

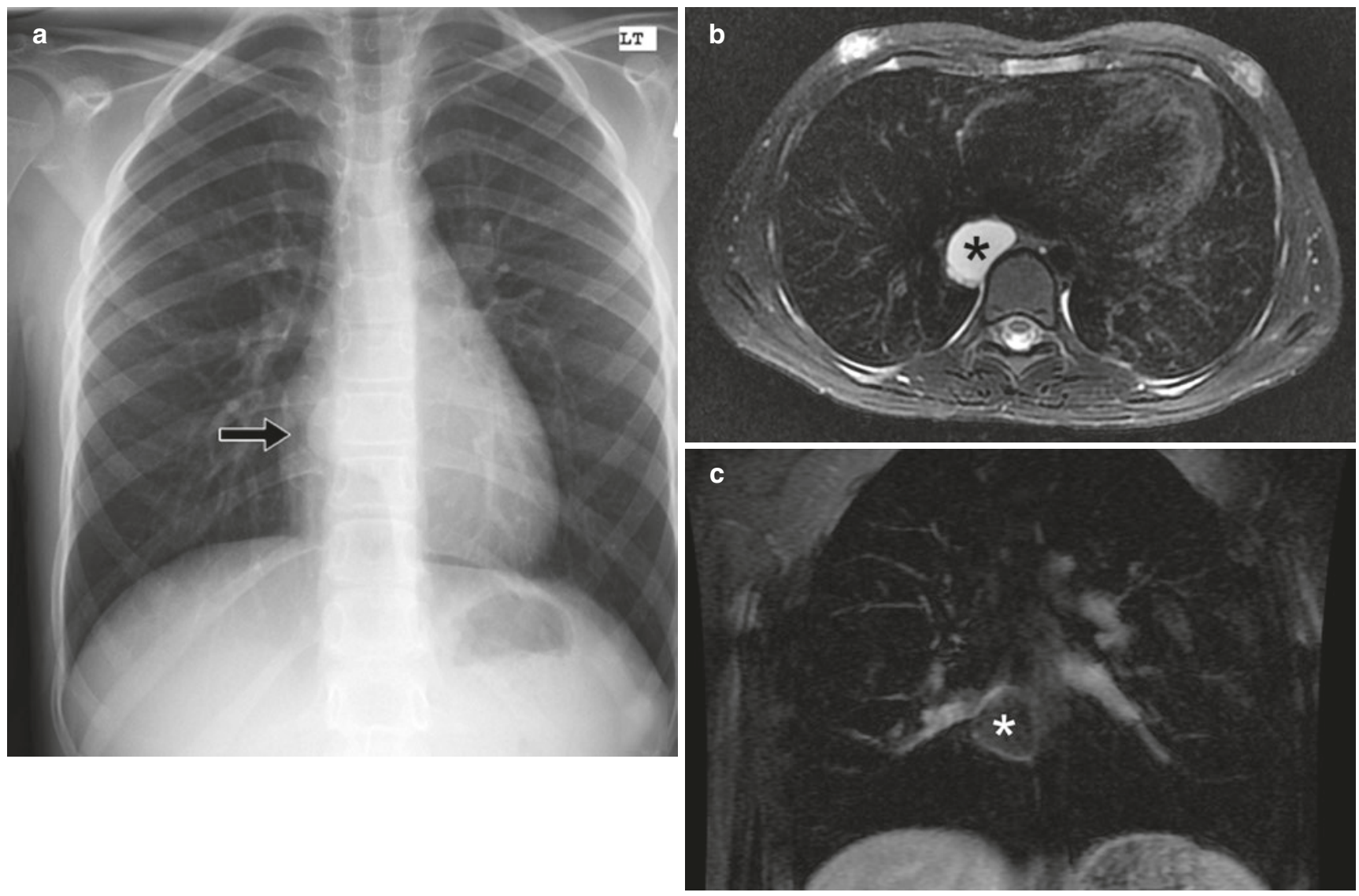

Fig. 10.15 A 14-year-old girl with esophageal duplication cyst who presented with dyspnea. The mediastinal mass was surgically excised and pathology was consistent with an esophageal duplication cyst. (a) Frontal chest radiograph demonstrates a well-circumscribed opacity (black arrow) overlying the right aspect of the heart. (b) Axial

Overall survival rate of thoracic neuroblastoma is superior to primary abdominal neuroblastoma with treatment usually involving a combination of surgical resection, chemotherapeutic agents, and radiation.

\section{Lymphatic Malformation}

Lymphatic malformation results from embryologic malformation of the lymphatic system. Such malformation results in lymph-containing smooth and skeletal muscle-lined channels with intervening fibro-connective tissue creating microcystic, macrocystic, or combined lesions of variable size. Intrathoracic lymphatic malformation in isolation is rare, accounting for less than $1 \%$ of all lymphatic malformations, and more generally results from extension of cervical, axillary, or mediastinal malformation. Typically present at birth, the majority of affected patients present to clinical attention before 2 years of age with an asymptomatic palpable mass although pain may occur with intralesional hemorrhage or
T2-weighted, fat-suppressed MR image shows a cystic lesion (asterisk) within the middle mediastinum. (c) Coronal enhanced T1-weighted, fat-suppressed MR image demonstrates a peripheral enhancement of the lesion (asterisk) within the right aspect of the middle mediastinum

infection. Large lesions, however, may produce symptoms such as dyspnea if there is secondary airway or vascular compression.

Intrathoracic lymphatic malformation, if small and asymptomatic, may be incidental findings on chest radiography performed for different indication. If located in the mediastinum, lymphatic malformation may present as a well-circumscribed mass or vague mediastinal fullness (Fig. 10.17a). If there is extension of the lymphatic malformation into the cervical region, deviation of the trachea may be identified on either the frontal or lateral radiography. MRI is currently considered the gold standard imaging modality in the evaluation of lymphatic malformations because it affords superior soft tissue contrast as compared with CT and enables evaluation of local extent and relationship to normal structures. As lymphatic malformations contain fluid, there is intrinsic T2 hyperintensity (Fig. 10.17b). Following contrast administration, there is variable enhancement of the 

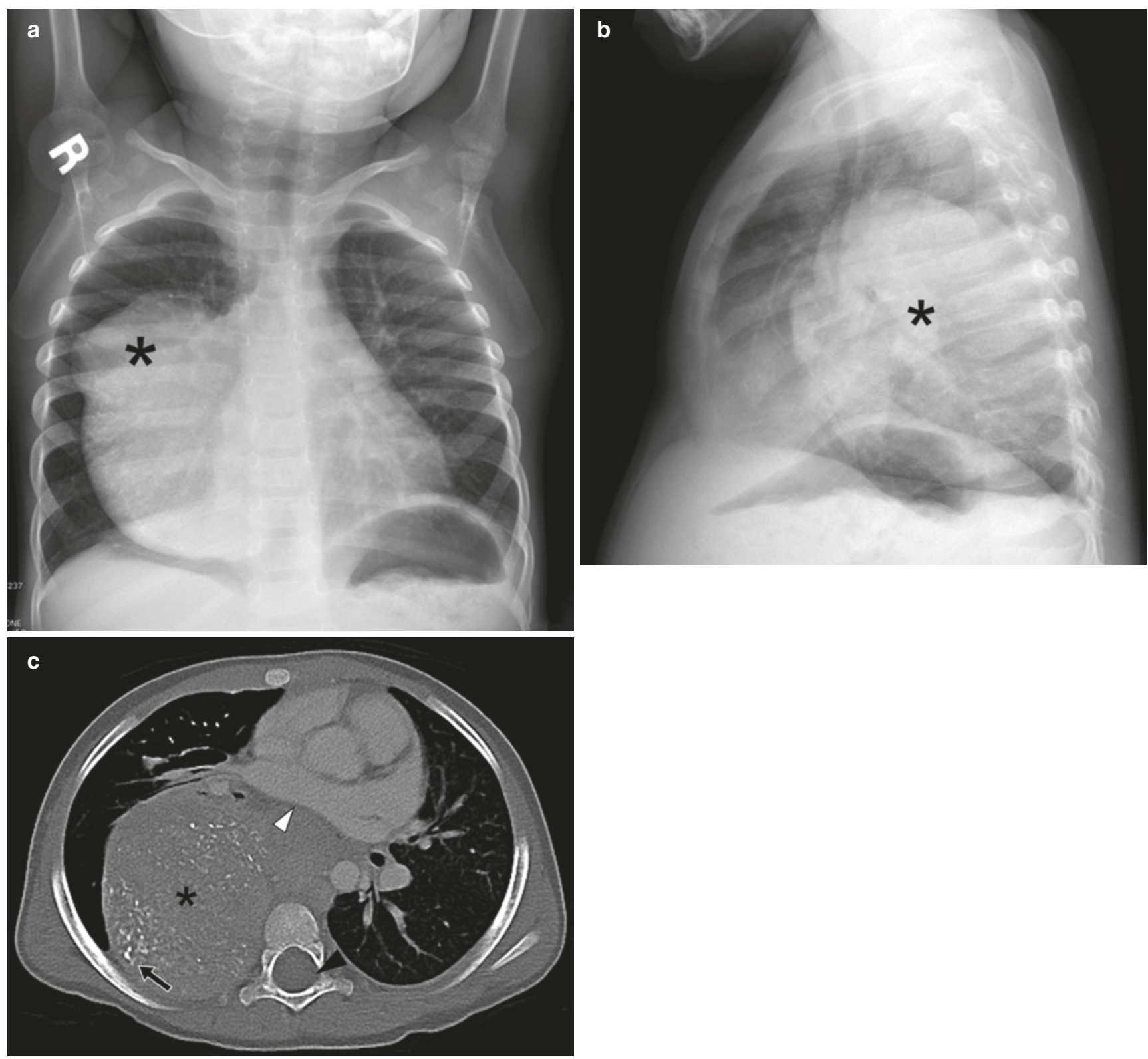

Fig. 10.16 A 2-year-old boy with thoracic neuroblastoma who presented with chest pain and paresthesia. (a) Frontal chest radiograph shows a well-circumscribed mass (asterisk) in the right hemithorax. Posterior mediastinal location of the mass is suspected by the presence of splaying of the right-sided posterior ribs. (b) Lateral chest radiograph confirms posterior mediastinal location of the mass (asterisk). (c) Axial enhanced bone window setting CT image demonstrates a large,

well-circumscribed mass (asterisk) within the posterior mediastinum with associated punctate calcifications (arrow). The mass displaces the heart (white arrowhead) anteriorly. Lack of normal rim of cerebrospinal fluid surrounding the spinal cord (black arrowhead) and amorphous soft tissue attenuation within the spinal canal raise concern for intracanalicular invasion 

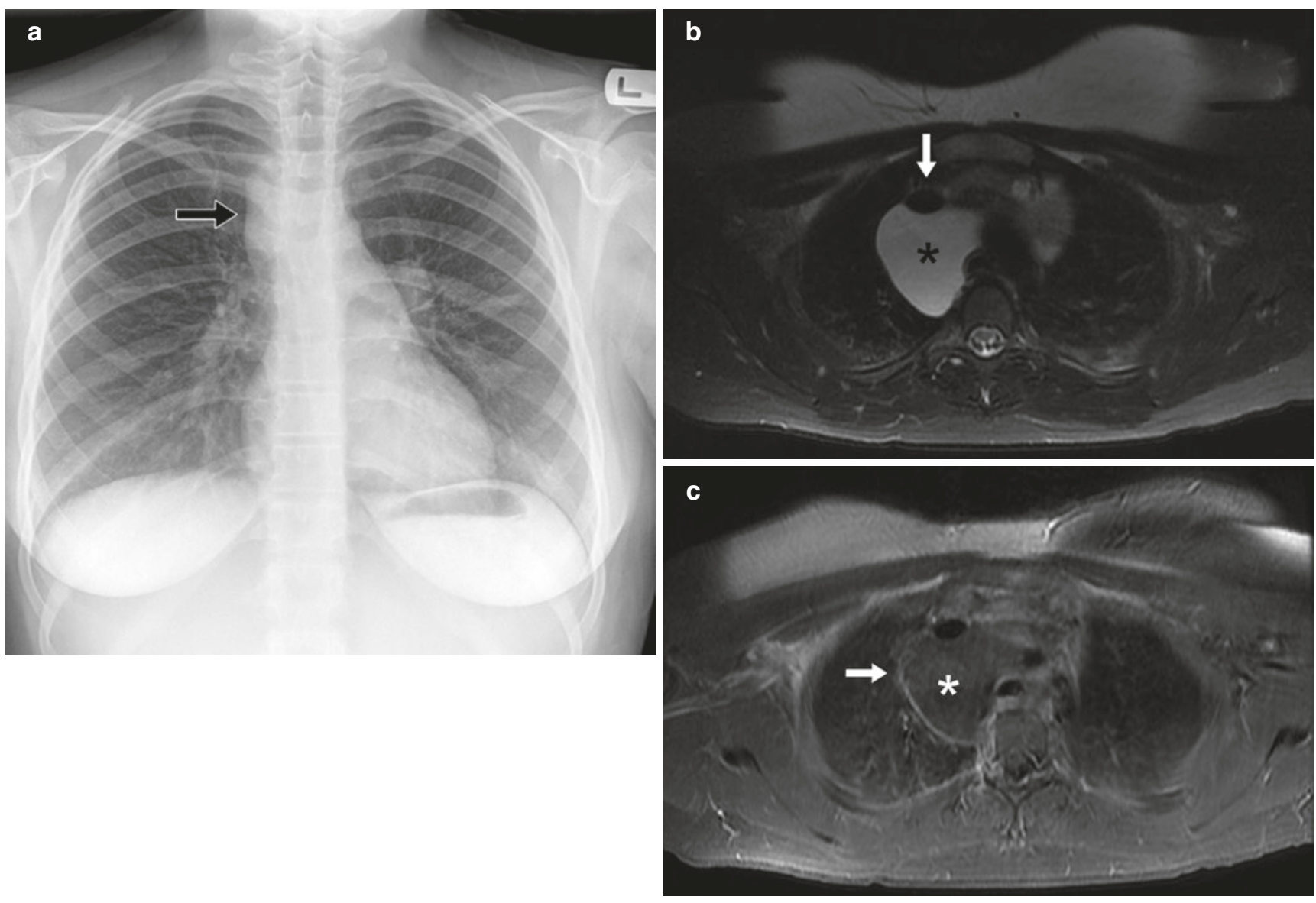

Fig. 10.17 A 14-year-old girl with thoracic lymphatic malformation who presented with cough. (a) Frontal chest radiograph shows prominence and undulating contour (arrow) of the right superior mediastinum. (b) Axial T2-weighted, fat-suppressed MR image demonstrates a

intervening septa and surrounding walls, which may increase in the setting of prior hemorrhage or infection (Fig. 10.17c).

Surgical resection and localized sclerotherapy are the primary forms of treatment for thoracic lymphatic malformations although complete surgical resection may be limited in extensive, infiltrative lymphatic malformations. With either therapy, relapse is common. Recently, given the success of topical therapies in the treatment of facial angiofibromas and the capillary vascular malformations associated with SturgeWeber syndrome, novel topical treatment modalities such as rapamycin have been described for lymphatic malformation with promising early results. cystic lesion (asterisk) located in the superior mediastinum posterior to the superior vena cava (arrow). (c) Axial enhanced T1-weighted, fatsuppressed MR image shows a peripheral enhancement (white arrow) of the lesion (asterisk) located within the superior mediastinum

\subsection{Conclusion}

Thoracic disorders mainly arise from lung parenchyma, large airway, or the mediastinum in the pediatric population. Imaging assessment plays an important role for initial detection, preoperative assessment for surgical lesions, and follow-up evaluation. Clear understanding of advantages and disadvantages of currently available imaging modalities as well as characteristic imaging findings of thoracic disorders has a great potential for optimal care of the pediatric patient. 


\section{Key Points}

- Chest radiography is the first-line and most commonly performed imaging examination in pediatric patients with potential thoracic disorders. CT provides superior characterization as compared with radiography though its use must be carefully weighed due to heighted susceptibility of pediatric patients to the harmful effects of ionizing radiation. Tailored acquisition parameters and image optimization techniques in accordance with the principal of ALARA must be used for dose reduction.

- Surfactant deficiency syndrome is the most common cause of death in premature infants, whereas transient tachypnea of the newborn is the most common cause of respiratory distress in full-term infants.

- Pneumonia is the most common cause of illness in childhood and is a significant cause of morbidity and mortality. Round pneumonia is a distinct manifestation of lower respiratory tract infection in children less than 8 years of age.

- Primary tracheomalacia secondary to prematurity and intrinsic weakness of the supporting cartilage is generally self-limiting and may require only supportive care in the first years of life.

- CT has an accuracy of close to $100 \%$ for the detection of aspirated foreign body and should be considered in the setting of high clinical suspicion with negative chest radiography.

- Neuroblastoma is the most common posterior mediastinal mass in pediatric patients and accounts for $90 \%$ of posterior mediastinal masses.

\section{Take-Home Messages}

- Differential considerations of commonly encountered pediatric chest radiology pathologies include both congenital conditions, such as surfactant deficiency syndrome due to prematurity and tracheomalacia, as well as acquired conditions, such as round pneumonia and neuroblastoma.

- Utilization of a three anatomic compartment model can help to narrow differential considerations in the imaging of pediatric chest pathologies.

- Chest radiography is the first-line and most commonly performed imaging examination in pediatric patients with potential thoracic disorders. The use of CT must be carefully weighed in pediatric chest radiology due to heighted susceptibility of pediatric patients to the harmful effects of ionizing radiation.

\section{References}

1. Sodhi KS, Lee EY. What all physicians should know about the potential radiation risk that computed tomography poses for paediatric patients. Acta Paediatr. 2014;103(8):807-11.

2. Sodhi KS, Krishna S, Saxena AK, Khandelwal N, Lee EY. Clinical application of "Justification" and "Optimization" principle of ALARA in pediatric CT imaging: "How many children can be protected from unnecessary radiation?". Eur J Radiol. 2015;84(9):1752-7.

3. Lee EY, Greenberg SB, Boiselle PM. Multidetector computed tomography of pediatric large airway diseases: state-of-the-art. Radiol Clin N Am. 2011;49(5):869-93.

4. Nogo AV, Winant AJ, Lee EY, Phillips GS. Strategies for reducing radiation dose in CT for pediatric patients: how we do it. Semin Roentgenol. 2018;53(2):124-31.

5. Ciet P, Tiddens HA, Wielopolski PA, Wild JM, Lee EY, Morana $\mathrm{G}$, Lequin MH. Magnetic resonance imaging in children: common problems and possible solutions for lung and airways imaging. Pediatr Radiol. 2015;45(13):1901-15.

6. Baez JC, Ciet P, Mulkern R, Seethamraju RT, Lee EY. Pediatric chest MR imaging: lung and airways. Magn Reson Imaging Clin N Am. 2015;23(2):337-49.

7. Sodhi KS, Khandelwal N, Saxena AK, Singh M, Agarwal R, Bhatia A, Lee EY. Rapid lung MRI in children with pulmonary infections: time to change our diagnostic algorithms. J Magn Reson Imaging. 2016;43(5):1196-206.

8. Liszewski MC, Gorkem S, Sodhi KS, Lee EY. Lung magnetic resonance imaging for pneumonia in children. Pediatr Radiol. 2017a;47(11):1420-30.

9. Agrons GA, Courtney SE, Stocker JT, Markowitz RI. Lung disease in premature neonates: radiologic-pathologic correlation. Radiographics. 2005;25:1047-73.

10. Liszewski MC, Lee EY. Neonatal lung disorders: pattern recognition approach to diagnosis. AJR Am J Roentgenol. 2018;210(5):964-75.

11. Edwards MO, Kotecha SJ, Kotecha S. Respiratory distress of the term newborn infant. Paediatr Respir Rev. 2013;14(1):29-36.

12. Liszewski MC, Stanescu AL, Phillips GS, Lee EY. Respiratory distress in neonates: underlying causes and current imaging assessment. Radiol Clin N Am. 2017b;55(4):629-44.

13. Dargaville PA, Copnell B. The epidemiology of meconium aspiration syndrome: incidence, risk factors, therapies, and outcome. Pediatrics. 2006;117(5):1712-21.

14. Winant AJ, Schooler GR, Concepcion NDP, Lee EY. Current updates on pediatric pulmonary infections. Semin Roentgenol. 2017;52(1):35-42.

15. Restrepo R, Rajaneeshankar P, Matapathi UM, Wu Y. Imaging of round pneumonia and mimics in children. Pediatr Radiol. 2010;40:1931-40.

16. Kim YW, Donnelly LF. Round pneumonia: imaging findings in a large series of children. Pediatr Radiol. 2007;37(12):1235-40.. Epub 2007 Oct 19.

17. Raol N, Metry D, Edmonds J, et al. Propranolol for the treatment of subglottic hemangiomas. Int J Pediatr Otorhinolaryngol. 2011;75:1510-4.

18. Haggstrom AN, et al. Clinical spectrum and risk of PHACE syndrome in cutaneous and airway hemangiomas. Arch Otolaryngol Head Neck Surg. 2011;137(7):680-7.

19. Zapala MA, Ho-Fung VM, Lee EY. Thoracic neoplasms in children: contemporary prospectives and imaging assessment. Radiol Clin N Am. 2017;55(4):657-76.

20. Liszewski MC, Ciet P, Sodhi KS, Lee EY. Updates on MRI evaluation of pediatric large airways. AJR Am J Roentgenol. 2017c;208(5):971-81.

21. Amini B, Huang SY, Tsai J, Benveniste MF, Robledo HH, Lee EY. Primary lung and large airway neoplasm in children: current 
imaging evaluation with multidetector computed tomography. Radiol Clin N Am. 2013;51(4):637-57.

22. Degnan AJ, Tocchio S, Kurtom W, Tadros SS. Pediatric neuroendocrine carcinoid tumors: management, pathology, and imaging findings in a pediatric referral center. Pediatr Blood Cancer. 2017;64(9).

23. Lee EY, Zucker EJ, Restrepo R, Daltro P, Boiselle PM. Advanced large airway CT imaging in children: evolution from axial to 4D assessment. Pediatr Radiol. 2013;43(3):285-97.

24. Tsai J, Lee EY, Restrepo R, Eisenberg RL. Focal large airway anomalies and abnormalities in pediatric patients. AJR Am J Roentgenol. 2013;201(2):W163-73.

25. Lee EY, Boiselle PM, Cleveland RH. Multidetector CT evaluation of congenital lung anomalies. Radiology. 2008a;247(3):632-48.

26. Lee EY, Litmanovich D, Boiselle PM. Multidetector CT evaluation of tracheobronchomalacia. Radiol Clin N Am. 2009;47(2):261-9.

27. Javia L, Harris MA, Fuller S. Rings, slings, and other tracheal disorders in the neonate. Semin Fetal Neonatal Med. 2016;21(4):277-84.

28. Lee EY, Mason KP, Zurakowski D, Waltz DA, Ralph A, Riaz F, Boiselle PM. MDCT assessment of tracheomalacia in symptomatic infants with mediastinal aortic vascular anomalies: preliminary technical experience. Pediatr Radiol. 2008b;38(1):82-8.

29. Shieh HF, Jennings RW. Three-dimensional printing of external airway splints for tracheomalacia. J Thorac Dis. 2017;9(3):414-6.

30. Green SS. Ingested and aspirated foreign bodies. Pediatr Rev. 2015;36(10):430-6.

31. Laya BF, Restrepo R, Lee EY. Practical imaging evaluation of foreign bodies in children: an update. Radiol Clin N Am. 2017;55(4):845-67.

32. Brian PS, Lim R, Avery LL. Review of ingested and aspirated foreign bodies in children and their clinical significance for radiologists. Radiographics. 2015;35(5):1528-38.

33. Wasilewska E, Lee EY, Esenberg RL. Unilateral hyperlucent lung in children. AJR Am J Roentgenol. 2012;198(5):W400-14.
34. Hegde SV, Hui PK, Lee EY. Tracheobronchial foreign bodies in children: imaging assessment. Semin Ultrasound CT MR. 2015;36(1):8-20.

35. Lee EY, Restrepo R, Dillman JR, Ridge CA, Boiselle PM. Imaging evaluation of pediatric trachea and bronchi: systematic review and updates. Semin Roentgenol. 2012;47(2):182-96.

36. Shin SM, Kim WS, Cheon JE, Jung AY, Youn BJ, Kim IO, Yeon KM. CT in children with suspected residual foreign body in airway after bronchoscopy. AJR Am J Roentgenol. 2009;192(6):1744-51.

37. Hellinger JC, Daubert M, Lee EY, Epleman M. Congenital thoracic vascular anomalies: evaluation with state-of-the-art MR imaging and MDCT. Radiol Clin N Am. 2011;49(5):969-96.

38. Etesami M, Ashwath R, Kanne J, Gilkeson RC, Rajiah P. Computed tomography in the evaluation of vascular rings and slings. Insights Imaging. 2014;5(4):507-21.

39. Hanneman K, Newman B, Chan F. Congenital variants and anomalies of the aortic arch. Radiographics. 2017;37(1):32-51.

40. Kondrachuk O, Yalynska T, Tammo R, Lee EY. Multidetector computed tomography evaluation of congenital mediastinal vascular anomalies in children. Semin Roentgenol. 2012;47(2):127-34.

41. Lee EY. Evaluation of non-vascular mediastinal masses in infants and children: an evidence-based practical approach. Pediatr Radiol. 2009;39(Suppl 2):S184-90.

42. Ranganath SH, Lee EY, Restrepo R, Eienberg RL. Mediastinal masses in children. AJR Am J Roentgenol. 2012;198(3):W197-216.

43. Hacker PG, Mahani MG, Heider A, Lee EY. Imaging evaluation of mediastinal masses in children and adults: practical diagnostic approach based on a new classification system. J Thorac Imaging. 2015;30(4):247-67.

44. Pavlus JD, Carter BW, Tolley MD, Keung ES, Khorashadi L, Lichtenberger JP. Imaging of thoracic neurogenic tumors. AJR Am J Roentgenol. 2016;207(3):552-61.

Open Access This chapter is licensed under the terms of the Creative Commons Attribution 4.0 International License (http://creativecommons. org/licenses/by/4.0/), which permits use, sharing, adaptation, distribution and reproduction in any medium or format, as long as you give appropriate credit to the original author(s) and the source, provide a link to the Creative Commons license and indicate if changes were made.

The images or other third party material in this chapter are included in the chapter's Creative Commons license, unless indicated otherwise in a credit line to the material. If material is not included in the chapter's Creative Commons license and your intended use is not permitted by statutory regulation or exceeds the permitted use, you will need to obtain permission directly from the copyright holder. 